TRANSACTIONS OF THE

AMERICAN MATHEMATICAL SOCIETY

Volume 360, Number 2, February 2008, Pages 1057-1088

S 0002-9947(07)04325-5

Article electronically published on July 23, 2007

\title{
THE KERNELS OF RADICAL HOMOMORPHISMS AND INTERSECTIONS OF PRIME IDEALS
}

\author{
HUNG LE PHAM
}

\begin{abstract}
We establish a necessary condition for a commutative Banach algebra $A$ so that there exists a homomorphism $\theta$ from $A$ into another Banach algebra such that the prime radical of the continuity ideal of $\theta$ is not a finite intersection of prime ideals in $A$. We prove that the prime radical of the continuity ideal of an epimorphism from $A$ onto another Banach algebra (or of a derivation from $A$ into a Banach $A$-bimodule) is always a finite intersection of prime ideals. Under an additional cardinality condition (and assuming the Continuum Hypothesis), this necessary condition is proved to be sufficient. En route, we give a general result on norming commutative semiprime algebras; extending the class of algebras known to be normable. We characterize those locally compact metrizable spaces $\Omega$ for which there exists a homomorphism from $\mathcal{C}_{0}(\Omega)$ into a radical Banach algebra whose kernel is not a finite intersection of prime ideals.
\end{abstract}

\section{INTRODUCTION}

Let $\theta: A \rightarrow B$ be a homomorphism from a commutative Banach algebra $A$ into a Banach algebra $B$. The continuity ideal of $\theta$ is defined to be the ideal

$$
\mathcal{I}(\theta)=\{a \in A \text { : the map } b \mapsto \theta(a b), A \rightarrow B \text {, is continuous }\} .
$$

We see that $\mathcal{I}(\theta)$ contains every ideal $I$ in $A$ on which $\theta$ is continuous. Let $\Omega$ be a locally compact space. In the case where $A=\mathcal{C}_{0}(\Omega)$, it is known that $\theta$ is continuous on $\mathcal{I}(\theta)$; however, this is not true in general.

In this paper, we shall give an answer to the following two equivalent questions:

A Is the kernel of a homomorphism from $\mathcal{C}_{0}(\Omega)$ into a radical Banach algebra always a finite intersection of prime ideals, for each non-compact locally compact space $\Omega$ ?

B Is the continuity ideal $\mathcal{I}(\theta)$ of a homomorphism $\theta$ from $\mathcal{C}_{0}(\Omega)$ into a Banach algebra always a finite intersection of prime ideals, for each locally compact space $\Omega$ ?

Denoted by $|\cdot|_{\Omega}$ the uniform norm on $\Omega$. It is a theorem of Kaplansky [19] that, for each (not necessarily complete) algebra norm $\|\cdot\|$ on $\mathcal{C}_{0}(\Omega)$ and each $f \in \mathcal{C}_{0}(\Omega)$, we have $\|f\| \geq|f|_{\Omega}$. This suggested a conjecture that each algebra norm on $\mathcal{C}_{0}(\Omega)$

Received by the editors June 1, 2005 and, in revised form, April 7, 2006.

2000 Mathematics Subject Classification. Primary 46H40, 46J10; Secondary 46J05, 13C05, $43 \mathrm{~A} 20$.

Key words and phrases. Banach algebra, algebra of continuous functions, automatic continuity, commutative algebra, prime ideal, locally compact space, locally compact group.

(C)2007 American Mathematical Society Reverts to public domain 28 years from publication 
is equivalent to $|\cdot|_{\Omega}$. The latter holds if and only if each homomorphism from $\mathcal{C}_{0}(\Omega)$ into a Banach algebra is continuous; this is known as the problem of Kaplansky.

In the 1970s, this long-standing open problem was resolved in the negative independently by Dales [5] and Esterle [10], [11, 12]. Moreover, they showed that, assuming the Continuum Hypothesis $(\mathrm{CH})$, for each non-compact locally compact space $\Omega$ and each non-modular prime ideal $P$ in $\mathcal{C}_{0}(\Omega)$ with $\left|\mathcal{C}_{0}(\Omega) / P\right|=\mathfrak{c}$, there exists a homomorphism from $\mathcal{C}_{0}(\Omega)$ into some radical Banach algebra with kernel precisely equal to $P$. For a full exposition of an improved and stronger form of this theorem, see [6].

Before this problem was resolved, it was proved by Bade and Curtis in 1 that each discontinuous homomorphism from $\mathcal{C}_{0}(\Omega)$ into a Banach algebra $B$ can be decomposed into a sum of a continuous homomorphism and a finite number of discontinuous linear maps, each of which is a homomorphism into the radical of $B$ when restricted to a maximal ideal of $\mathcal{C}_{0}(\Omega)$ (this result was later improved in [10], [18, and 23]). This important result sheds light on the structure of discontinuous homomorphisms from $\mathcal{C}_{0}(\Omega)$. We summarize below part of the result that is more relevant to our discussion; here $\Omega^{b}$ denotes the one-point compactification of $\Omega$, and the ideals $J_{p}$ and $M_{p}$, for $p \in \Omega^{b}$, are to be defined in the next section.

Theorem 1.1. Let $\Omega$ be a non-empty locally compact space, and let $\theta$ be a homomorphism from $\mathcal{C}_{0}(\Omega)$ into a Banach algebra $B$.

(i) The continuity ideal $\mathcal{I}(\theta)$ is the largest ideal of $\mathcal{C}_{0}(\Omega)$ on which $\theta$ is continuous.

(ii) There exists a finite subset $\left\{p_{1}, \ldots, p_{n}\right\}$ of $\Omega^{b}$ such that

$$
\bigcap_{i=1}^{n} J_{p_{i}} \subset \mathcal{I}(\theta) \subset \bigcap_{i=1}^{n} M_{p_{i}} .
$$

(iii) There exists a homomorphism $\mu$ from $\bigcap_{i=1}^{n} M_{p_{i}}$ into $\operatorname{rad} B$ such that $\mathcal{I}(\theta)$ $=\operatorname{ker} \mu$.

(iv) The ideals $\operatorname{ker} \theta$ and $\mathcal{I}(\theta)$ are always intersections of prime ideals.

(v) In the case where $B$ is radical, $\mathcal{I}(\theta)=\operatorname{ker} \theta$.

From the theorem of Dales and of Esterle, it follows easily that, assuming $\mathrm{CH}$, each ideal which is a finite intersection of non-modular prime ideals in $\mathcal{C}_{0}(\Omega)$ (with a suitable cardinality condition) is the kernel of a homomorphism from $\mathcal{C}_{0}(\Omega)$ into a radical Banach algebra. It was immediately questioned if the converse is true; this is Question A. By Theorem 1.1 (iii) and (v), this is equivalent to Question B. This same theorem, particularly parts (ii) and (iv), also suggests that the answer to the questions should be "yes" for every space $\Omega$. If the answers were "yes", we would have, in some sense, a complete picture of the structure of homomorphisms from $\mathcal{C}_{0}(\Omega)$ (at least in the case where $\left|\mathcal{C}_{0}(\Omega)\right|=\mathfrak{c}$ ).

These questions were first raised in [4, 10]. Question B was partially answered (in the affirmative) in 10 in the cases where $\Omega$ is a compact F-space, and where $\Omega$ is compact and $\beta(\Omega \backslash\{p\})$ is an F-space for each non-P-point $p$ of $\Omega$ (thus implying an affirmative answer for the spaces $\mathbb{N}, \beta \mathbb{N}$, and $\beta \mathbb{N} \backslash \mathbb{N}$ ). There are further partial answers in [7]: here the authors restrict to ideals of the form $J_{p}$. However, the questions were left open for almost all spaces. 
To tackle Question A (and B) we first consider a more general version of Question $B$. We shall reduce the general question of whether or not there exists a homomorphism $\theta$ from a given commutative Banach algebra $A$ into another Banach algebra such that the prime radical of $\mathcal{I}(\theta)$ cannot be represented as a finite intersection of prime ideals to an intrinsic question about the structure of the prime ideals of $A$. We shall then use this reduction to prove that the prime radical of the continuity ideal of an epimorphism from $A$ onto another Banach algebra (or of a derivation from $A$ into a Banach $A$-bimodule) is always a finite intersection of prime ideals in $A$ (\$4).

A little offtrack of our main discussion, and in order to show that it is necessary, in the above reduction, to consider the prime radicals of the continuity ideals, we shall construct homomorphisms of various types between commutative Banach algebras whose continuity ideals are not intersections of prime ideals. In particular, we shall construct a derivation $\mathcal{D}: \mathcal{A} \rightarrow \mathcal{A}^{\prime}$, where $\mathcal{A}=A\left(\overline{\mathbb{D}}^{2}\right)$, such that the continuity ideal of $\mathcal{D}$ is not an intersection of prime ideals in $\mathcal{A}(95)$.

In another direction, Esterle 13 generalized the above-mentioned construction to a construction of a homomorphism from an arbitrary commutative algebra $A$ into some radical Banach algebra, with the kernel to be any prescribed non-modular prime ideal $P$ in $A$ such that $|A / P|=\mathfrak{c}$. (See [9, Chapter 5] for a different approach to this result, which will be sketched very briefly at the beginning of 96 .) Again, this easily implies the same result for finite intersections of prime ideals. We shall extend the result to intersections of various infinite families of prime ideals, thus enlarging the class of commutative algebras which are known to be normable (\$7). The precise statement of this is as follows; we refer the reader to $\$$ for the definition of 'pseudo-finiteness' and of 'intersection non-redundancy'.

Theorem $(\mathrm{CH})$. Let $A$ be a commutative algebra, and let $I$ be the intersection of a pseudo-finite family of non-modular prime ideals. Suppose that $|A / I|=\mathfrak{c}$. Then A/I can be embedded into a radical Banach algebra.

The above extension yields a partial converse of the previously mentioned reduction. In summary, we shall prove the following result (Theorems 4.5 and 7.4).

Theorem. Let $A$ be a commutative Banach algebra.

(i) Suppose that there exists a homomorphism $\theta$ from A into another Banach algebra such that the prime radical of $\mathcal{I}(\theta)$ is not a finite intersection of prime ideals. Then there exists an intersection non-redundant pseudofinite sequence of non-closed prime ideals in A.

(ii) $(\mathrm{CH})$ Suppose that $I$ is the intersection of an intersection non-redundant pseudo-finite sequence of non-closed prime ideals in A. Suppose further that $|A / I|=\mathfrak{c}$. Then there exists a homomorphism $\theta$ from $A$ into another Banach algebra with continuity ideal equal to $I$ - a semiprime ideal, but not a finite intersection of prime ideals in $A$.

We shall then apply the above results to Questions A and B. We give the following characterizations (Theorems 8.8 and 9.5 . cf. Theorem 8.1).

Theorem (A). Let $\Omega$ be a locally compact metrizable space, and let $p$ denote the point adjoined to $\Omega$ to obtain $\Omega^{b}$. 
(i) Suppose that $p \notin \partial^{(\infty)}\left(\Omega^{b}\right)$. Then the kernel of each homomorphism from $\mathcal{C}_{0}(\Omega)$ into a radical Banach algebra is a finite intersection of non-modular prime ideals.

(ii) (CH) Suppose that $p \in \partial^{(\infty)}\left(\Omega^{b}\right)$. Then there exists a homomorphism $\theta$ from $\mathcal{C}_{0}(\Omega)$ into a radical Banach algebra such that the kernel of $\theta$ is not a finite intersection of prime ideals.

Theorem (B). Let $\Omega$ be a locally compact metrizable space.

(i) Suppose that $\Omega^{b}$ has finite limit level. Then the continuity ideal $\mathcal{I}(\theta)$ of each homomorphism $\theta$ from $\mathcal{C}_{0}(\Omega)$ into another Banach algebra is a finite intersection of prime ideals.

(ii) $(\mathrm{CH})$ Suppose that $\Omega^{b}$ has infinite limit level. Then there exists a homomorphism $\theta$ from $\mathcal{C}_{0}(\Omega)$ into a Banach algebra such that the continuity ideal of $\theta$ is not a finite intersection of prime ideals.

Thus, for example, assuming $\mathrm{CH}$, there exists a homomorphism $\theta$ from $\mathcal{C}_{0}(\mathbb{R})$ into a radical Banach algebra such that $\operatorname{ker} \theta$ is not a finite intersection of prime ideals. This answers Questions A and B in the negative.

Finally, we shall characterize those locally compact groups (not necessarily metrizable) $G$ such that there exists a homomorphism $\theta$ from $\mathcal{C}_{0}(G)$ into a radical Banach algebra (respectively, a Banach algebra) such that the kernel (respectively, the continuity ideal) of $\theta$ is not a finite intersection of prime ideals. We also give an application to prime ideals in Abelian group algebras (\$10).

Remark. We note here two other equivalent formulations of Questions A and B.

C Is the kernel of a homomorphism from $\mathcal{C}_{0}(\Omega)$ into a Banach algebra always the intersection of a closed ideal and a finite number of prime ideals, for each locally compact space $\Omega$ ?

Next, let $\theta$ be a homomorphism from $\mathcal{C}_{0}(\Omega)$ into a Banach algebra. Then $q(f)=$ $\|\theta(f)\|\left(f \in \mathcal{C}_{0}(\Omega)\right)$ is an algebra seminorm on $\mathcal{C}_{0}(\Omega)$. It is proved in [10] that $\mathcal{I}(\theta)$ is the intersection of the $q$-closed prime ideals in $\mathcal{C}_{0}(\Omega)$, and that each chain of such prime ideals are well-ordered (with respect to the inclusion). Questions A, B are also equivalent to the following:

D Is the set of prime ideals which are closed with respect to a seminorm on $\mathcal{C}_{0}(\Omega)$ always a finite union of well-ordered chains, for each locally compact space $\Omega$ ?

In this paper, we shall work explicitly with Questions A and B only; the corresponding results for $\mathrm{C}$ and $\mathrm{D}$ can be derived easily.

Remark. In all the constructions mentioned above, the Continuum Hypothesis is indispensable, for it has been proved by Solovay and Woodin that it is relatively consistent with ZFC that all homomorphisms from each algebra $\mathcal{C}_{0}(\Omega)$ into a Banach algebra are continuous (see [8] for the proof and references).

\section{Preliminary definitions and notations}

In this paper, all the results that require the Continuum Hypothesis will be marked by the abbreviation $\mathrm{CH}$. All our algebras, fields, and integral domains are over the complex field $\mathbb{C}$.

Let $A$ be a commutative algebra. The (conditional) unitization $A^{\#}$ of $A$ is defined as the algebra $A$ itself if $A$ is unital, and as $A$ with identity adjoined 
otherwise. The identity of $A^{\#}$ is denoted by either $\mathbf{e}_{A}$ or simply 1 . For a subset $S$ of $A$, the smallest subalgebra of $A$ containing $S$ is denoted by alg $S$.

A prime ideal or semiprime ideal in $A$ must be a proper ideal. However, we consider $A$ itself as a finite intersection of prime ideals (the intersection of the empty collection of prime ideals).

Define the prime radical $\sqrt{I}$ of an ideal $I$ in $A$ to be the intersection of all prime ideals in $A$ containing $I$, so that

$$
\sqrt{I}=\left\{a \in A: a^{n} \in I \text { for some } n \in \mathbb{N}\right\} .
$$

For each ideal $I$ in $A$ and each element $a \in A^{\#}$, define the quotient of $I$ by $a$ to be the ideal

$$
I: a=\{b \in A: a b \in I\} .
$$

Clearly we have $I \subset I: a$ in each case. Note that, when $A$ is a topological algebra and $I$ is a closed ideal, the ideal $I: a$ is also closed for each $a \in A^{\#}$.

Let $I$ be an ideal in $A^{\#}$. A subset $S$ of $A^{\#}$ is algebraically independent modulo $I$ if $p\left(a_{1}, \ldots, a_{n}\right) \notin I$ for each $n \in \mathbb{N}$, each non-zero polynomial $p \in \mathbb{C}\left[X_{1}, \ldots, X_{n}\right]$, and each $n$-tuple $\left(a_{1}, \ldots, a_{n}\right)$ of distinct elements of $S$. A transcendence basis for $A^{\#}$ modulo $I$ is a maximal set among all the subsets of $A^{\#}$ which are algebraically independent modulo $I$; such a basis always exists.

Next, we are going to present some standard automatic continuity theory; for details, see 6] or [24].

Let $T: E \rightarrow F$ be a linear operator from a Banach space $E$ into a Banach space $F$. The separating space of $T$, denoted by $\mathfrak{S}(T)$, is defined as

$$
\left\{v \in F \text { : there exists a sequence }\left(u_{n}\right) \subset E \text { with } u_{n} \rightarrow 0 \text { and } T u_{n} \rightarrow v\right\} \text {. }
$$

The space $\mathfrak{S}(T)$ is a closed subspace of $F$, and, by the closed graph theorem, $T$ is continuous if and only if $\mathfrak{S}(T)=\{0\}$. In fact, let $S: F \rightarrow G$ be a bounded linear operator from $F$ into another Banach space $G$. Then $\mathfrak{S}(S T)=\overline{S(\mathfrak{S}(T))}$, and $S T$ is continuous if and only if $S(\mathfrak{S}(T))=\{0\}$; see [6, 5.2.2] or [24, 1.3].

Now let $\theta: A \rightarrow B$ be a homomorphism from a commutative Banach algebra $A$ into a Banach algebra $B$. Then $\mathfrak{S}(\theta)$ is a closed ideal in $\overline{\theta(A)}$. By the previous paragraph, we have

$$
\mathcal{I}(\theta)=\{a \in A: \theta(a) \mathfrak{S}(\theta)=\{0\}\} .
$$

We have the following famous stability lemma (homomorphism version); see [6, 5.2.7] or [24, 1.6] for the statement and proof of a more general version.

Proposition 2.1 (Stability lemma). Let $\theta$ be a homomorphism from a commutative Banach algebra $A$ into a Banach algebra $B$. Let $\left(a_{n}: n \in \mathbb{N}\right)$ be a sequence in $A$. Then there is an integer $N \in \mathbb{N}$ such that

$$
\overline{\theta\left(a_{1} \cdots a_{n}\right) \mathfrak{S}(\theta)}=\overline{\theta\left(a_{1} \cdots a_{m}\right) \mathfrak{S}(\theta)} \quad(m, n \geq N) .
$$

Let $A$ be a Banach algebra, and let $M$ be a Banach $A$-bimodule. We adopt the convention that the norm on $M$ satisfies

$$
\|a \cdot x\| \leq\|a\|\|x\| \quad \text { and } \quad\|x \cdot a\| \leq\|a\|\|x\| \quad(a \in A, x \in M) ;
$$

if this is not the case, we can always replace the given norm on $M$ by another, equivalent, norm such that these inequalities hold. 
The Banach dual module of $M$ is the Banach dual space of $M$, denoted by $M^{\prime}$, with the left and right $A$-module actions on $M^{\prime}$ being the dual actions of those on $M$, that is,

$$
(\lambda \cdot a)(x)=\lambda(a \cdot x) \quad \text { and } \quad(a \cdot \lambda)(x)=\lambda(x \cdot a) \quad\left(a \in A, \lambda \in M^{\prime}, x \in M\right) .
$$

Then $M^{\prime}$ is indeed a Banach $A$-bimodule.

Let $D$ be a derivation from a commutative Banach algebra $A$ into a Banach $A$-bimodule $M$. The continuity ideal of $D$ is defined to be

$$
\mathcal{I}(D)=\{a \in A: a \cdot \mathfrak{S}(D)=\{0\}\} .
$$

Again, we have

$$
\mathcal{I}(D)=\{a \in A: \text { the map } b \mapsto D(a b), A \rightarrow M, \text { is continuous }\} .
$$

We see that $\mathcal{I}(D)$ is a closed ideal in $A$. The continuity ideals of derivations are actually "special cases" of those of homomorphisms. Indeed, define a Banach algebra $B$ to be the direct sum $A \oplus M$ with $\ell^{1}$-norm, so that

$$
\|a \oplus x\|=\|a\|+\|x\| \quad(a \in A, x \in M) .
$$

The product on $B$ is given by

$$
(a \oplus x)(b \oplus y)=a b \oplus(a \cdot y+x \cdot b) \quad(a, b \in A, x, y \in M) .
$$

Define a homomorphism $\theta: A \rightarrow B$ by setting $\theta(a)=a \oplus D a$. Then $\mathcal{I}(\theta)=\mathcal{I}(D)$.

For a discussion of the theory of the algebras of continuous functions, see [6], 9 ] or [15. Here, we just give some facts which are needed in our discussion.

Let $\Omega$ be a locally compact space; the convention is that locally compact spaces and compact spaces are Hausdorff. The Stone-Čech compactification of $\Omega$ is denoted by $\beta \Omega$. Denote by $\mathcal{C}_{c}(\Omega)$ the algebra of compactly supported continuous functions on $\Omega$. For each $p \in \Omega$, define

$$
\begin{aligned}
J_{p}^{\Omega} & =\left\{f \in \mathcal{C}_{0}(\Omega): f \text { is zero on a neighbourhood of } p\right\}, \\
M_{p}^{\Omega} & =\left\{f \in \mathcal{C}_{0}(\Omega): f(p)=0\right\} .
\end{aligned}
$$

We shall omit the superscript $\Omega$ and write $J_{p}$ and $M_{p}$ when there is no ambiguity. For $p$ being the point (at infinity) adjoined to $\Omega$ to obtain $\Omega^{b}$, we also set

$$
J_{p}=\mathcal{C}_{c}(\Omega) \text { and } M_{p}=\mathcal{C}_{0}(\Omega) .
$$

For each prime ideal $P$ in $\mathcal{C}_{0}(\Omega)$, there always exists a unique point $p \in \Omega^{b}$ such that $J_{p} \subset P \subset M_{p}$; we say that $P$ is supported at the point $p$. It can be seen that $P$ is modular if and only if its support point belongs to $\Omega$.

It is an important fact that, for each prime ideal $P$ in $\mathcal{C}_{0}(\Omega)$, the set of prime ideals in $\mathcal{C}_{0}(\Omega)$ which contain $P$ is a chain with respect to the inclusion relation. Thus, for each $n \in \mathbb{N}$, a semiprime ideal in $\mathcal{C}_{0}(\Omega)$ that contains an intersection of $n$ prime ideals is itself an intersection of $n$ prime ideals.

For each function $f$ continuous on $\Omega$, the zero set of $f$ is denoted by $\mathbf{Z}_{\Omega}(f)$ or $\mathbf{Z}(f)$. The set of zero sets of continuous functions on $\Omega$ is denoted by $\mathbf{Z}[\Omega]$. For each closed subset $Z \subset \Omega$, we have $Z=\mathbf{Z}(f)$ for some function $f \in \mathcal{C}_{0}(\Omega)$ if and only if $\Omega \backslash Z$ is $\sigma$-compact.

A $z$-filter $\mathcal{F}$ on $\Omega$ is a non-empty proper subset of $\mathbf{Z}[\Omega]$ satisfying:

(i) $Z_{1} \cap Z_{2}$ belongs to $\mathcal{F}$ whenever both $Z_{1}$ and $Z_{2}$ belong to $\mathcal{F}$,

(ii) if $Z_{1} \in \mathcal{F}, Z_{2} \in \mathbf{Z}[\Omega]$ and $Z_{1} \subset Z_{2}$, then $Z_{2}$ also belongs to $\mathcal{F}$. 
Each $z$-filter $\mathcal{F}$ corresponds to an ideal

$$
\{f \in \mathcal{C}(\Omega): \mathbf{Z}(f) \in \mathcal{F}\}
$$

denoted by $\mathbf{Z}_{\Omega}^{-1}[\mathcal{F}]$ or $\mathbf{Z}^{-1}[\mathcal{F}]$; each such ideal is called a $z$-ideal. A $z$-filter $\mathcal{F}$ is a prime $z$-filter if $Z_{1} \cup Z_{2} \notin \mathcal{F}$ whenever $Z_{1}, Z_{2} \in \mathbf{Z}[\Omega] \backslash \mathcal{F}$. A maximal $z$-filter is called a $z$-ultrafilter. A $z$-ultrafilter is always prime.

Finally, let $\Omega$ be a compact space. Define $\partial \Omega$ to be the set of all limit points of $\Omega$, i.e., $\Omega \backslash \partial \Omega$ is the set of isolated points of $\Omega$. Then $\partial \Omega$ is a compact subset of $\Omega$, called the derived set of $\Omega$. Since $\Omega$ is compact, $\partial \Omega$ is non-empty unless $\Omega$ is finite. We define a non-increasing sequence $\left(\partial^{(n)} \Omega: n \in \mathbb{Z}^{+}\right)$of compact subsets of $\Omega$ as follows:

(i) put $\partial^{(0)} \Omega=\Omega$;

(ii) for each $n \in \mathbb{Z}^{+}$, define $\partial^{(n+1)} \Omega=\partial\left(\partial^{(n)} \Omega\right)$.

Define $\partial^{(\infty)} \Omega=\bigcap\left\{\partial^{(n)} \Omega: n \in \mathbb{Z}^{+}\right\}$. By the compactness, either $\partial^{(\infty)} \Omega$ is nonempty or $\partial^{(l)} \Omega$ is empty for some $l \in \mathbb{Z}^{+}$. In the former case, we say that $\Omega$ has infinite limit level; in the latter, we say that $\Omega$ has finite limit level.

\section{Pseudo-Finite families of PRIme ideAls}

Let $\left(E_{\alpha}: \alpha \in S\right)$ be a family of subsets of a given set $E$ indexed by another set $S$. The following concepts play an important role in the sequel; we shall see (in \$4) how these concepts arise naturally in our problem.

Definition 3.1. The family $\left(E_{\alpha}: \alpha \in S\right)$ is pseudo-finite if $a \in E_{\alpha}$ for all but finitely many $\alpha \in S$ whenever $a \in \bigcup_{\alpha \in S} E_{\alpha}$.

Certainly, each finite family is pseudo-finite.

Definition 3.2. The family $\left(E_{\alpha}: \alpha \in S\right)$ is intersection-redundant if there exists a subset $T$ of $S$ such that $T \neq S$ and such that

$$
\bigcap_{\alpha \in T} E_{\alpha}=\bigcap_{\alpha \in S} E_{\alpha}
$$

Otherwise, we say that the family is intersection non-redundant.

Now let $A$ be a commutative algebra. Note that, for each infinite pseudo-finite family of prime ideals $\left(P_{\alpha}: \alpha \in S\right)$ in $A$, the union $\bigcup_{\alpha \in S} P_{\alpha}$ must be an ideal and hence it is either prime in $A$ or $A$ itself.

First, we shall present some rather interesting properties which show, to an extent, the similarity between pseudo-finite and finite families of prime ideals.

Lemma 3.3. Let $A$ be a commutative algebra, and let $P$ be a prime ideal in $A$. Suppose that $\left(I_{\alpha}: \alpha \in S\right)$ is a pseudo-finite family of ideals in $A$ such that $\bigcap_{\alpha \in S} I_{\alpha} \subset P$. Then $I_{\alpha_{0}} \subset P$ for some $\alpha_{0} \in S$.

Proof. Assume toward a contradiction that $I_{\alpha} \not \subset P(\alpha \in S)$. Choose $a_{0} \in I_{\alpha_{0}} \backslash P$ for some $\alpha_{0} \in S$. Then, by the pseudo-finiteness, we have $a_{0} \in I_{\alpha}$ for all but finitely many $\alpha \in S$. Let $\alpha_{1}, \ldots, \alpha_{n}$ be the indices $\alpha \in S$ such that $a_{0} \notin I_{\alpha}$. For each $k \in \mathbb{N}$ with $1 \leq k \leq n$, choose $a_{k} \in I_{\alpha_{k}} \backslash P$, and set $a=a_{0} a_{1} \cdots a_{n}$. Then $a \in I_{\alpha}(\alpha \in S)$, but $a \notin P$, by the primeness of $P$; a contradiction to the fact that $\bigcap_{\alpha \in S} I_{\alpha} \subset P$. 
Lemma 3.4. Let $\left(P_{\alpha}: \alpha \in S\right)$ be a pseudo-finite family of prime ideals in a commutative algebra $A$. Then the following are equivalent:

(a) $\left(P_{\alpha}\right)$ is intersection non-redundant;

(b) $P_{\alpha} \not \subset P_{\beta} \quad(\alpha \neq \beta \in S)$;

(c) $\bigcap_{\beta \neq \alpha} P_{\beta} \not \subset P_{\alpha}$ for each $\alpha \in S$.

Proof. This follows from the previous lemma.

Lemma 3.5. Let $\left(P_{\alpha}: \alpha \in S\right)$ be a non-empty pseudo-finite family of prime ideals in a commutative algebra $A$. Let $\left(P_{\alpha}: \alpha \in T\right)$ be the subfamily of distinct minimal elements of $\left(P_{\alpha}: \alpha \in S\right)$. Then:

(i) $\left(P_{\alpha}: \alpha \in T\right)$ is intersection non-redundant, and $\bigcap_{\alpha \in S} P_{\alpha}=\bigcap_{\alpha \in T} P_{\alpha}$;

(ii) the ideal $\bigcap_{\alpha \in S} P_{\alpha}$ cannot be represented as an intersection of strictly fewer than $|T|$ prime ideals.

In particular, $T$ is non-empty.

Proof. (i) Obviously $\left(P_{\alpha}: \alpha \in T\right)$ satisfies Lemma 3.4(b), so this family is intersection non-redundant. Since $\left(P_{\alpha}: \alpha \in S\right)$ is pseudo-finite, each $P_{\alpha}(\alpha \in S)$ must contain a minimal element $P_{\beta}$ for some $\beta \in T$. Thus $\bigcap_{\alpha \in S} P_{\alpha}=\bigcap_{\alpha \in T} P_{\alpha}$.

(ii) Set $I=\bigcap_{\alpha \in S} P_{\alpha}=\bigcap_{\alpha \in T} P_{\alpha}$. By Lemma 3.4, we can choose

$$
a_{\alpha} \in\left(\bigcap_{\beta \neq \alpha, \beta \in T} P_{\beta}\right) \backslash P_{\alpha} \quad \text { for each } \alpha \in T .
$$

We see that $a_{\alpha} a_{\beta} \in I$ for $\alpha \neq \beta \in T$. It follows easily that $I$ cannot be represented as an intersection of strictly fewer than $|T|$ prime ideals.

Lemma 3.6. Let $A$ be a commutative Banach algebra. Let $\left(P_{\alpha}: \alpha \in S\right)$ be an intersection non-redundant pseudo-finite family of prime ideals in $A$. Then the number of closed prime ideals in the family $\left(P_{\alpha}\right)$ is finite.

Proof. Assume towards a contradiction that there exist distinct closed prime ideals $P_{\alpha_{n}}(n \in \mathbb{N})$ in the family $\left(P_{\alpha}: \alpha \in S\right)$. By Lemma 3.4 for each $n \in \mathbb{N}$, we can choose $a_{n} \in\left(\bigcap_{i \neq n} P_{\alpha_{i}}\right) \backslash P_{\alpha_{n}}$; we can further suppose that $\left\|a_{n}\right\| \leq 1 / 2^{n}$. Set $a=\sum_{n=2}^{\infty} a_{n}$. Then $a \in P_{\alpha_{1}}$. But then, by pseudo-finiteness, we have $a \in P_{\alpha_{k}}$ for some $k \geq 2$. However, we have $a-a_{k}=\sum\left\{a_{n}: n \notin\{1, k\}\right\} \in P_{\alpha_{k}}$, so that $a_{k} \in P_{\alpha_{k}}$, contradicting the choice of $a_{k}$.

Example 3.7. Let $S$ be a non-empty index set, and let $X_{\alpha}$ be an indeterminate for each $\alpha \in S$. For $n \in \mathbb{N}$, for $\alpha_{1}, \ldots, \alpha_{n}$ in $S$, and for $k_{1}, \ldots, k_{n} \in \mathbb{N}$, the formal product $X_{\alpha_{1}}^{k_{1}} \cdots X_{\alpha_{n}}^{k_{n}}$ is called a monomial. A formal power series of indeterminates $X_{\alpha}(\alpha \in S)$ is a formal series of the form $f=\lambda_{0}+\sum_{n=1}^{\infty} \lambda_{n} M_{n}$, where $\lambda_{n} \in \mathbb{C}$ and $M_{n}$ is a monomial (for each $n$ ). We identify, add, and multiply two formal power series according to the "standard" rules (similar to those for polynomials). The resulting algebra is called the formal power series algebra over $S$, and is denoted by $\mathfrak{F}_{S}$. It is easily seen that $\mathfrak{F}_{S}$ is an integral domain.

For each $\alpha \in S$, let $Q_{\alpha}$ be the ideal in $\mathfrak{F}_{S}$ generated by $\left\{X_{\beta}: \beta \in S, \beta \neq \alpha\right\}$. We can check that $Q_{\alpha}$ is a prime ideal in $\mathfrak{F}_{S}$. Thus $\left(Q_{\alpha}: \alpha \in S\right)$ is a pseudo-finite family of prime ideals, and $\left(Q_{\alpha}: \alpha \in S\right)$ is intersection non-redundant. 
Example 3.8. Now let $\mathcal{A}_{S}$ be the subalgebra of $\mathfrak{F}_{S}$ that consists of elements $f$ which can be represented as a series $\lambda_{0}+\sum_{n=1}^{\infty} \lambda_{n} M_{n}$ with

$$
\|f\|_{1}=\sum_{n=0}^{\infty}\left|\lambda_{n}\right|<\infty
$$

where $\left(\lambda_{n}\right) \subset \mathbb{C}\left(n \in \mathbb{Z}^{+}\right)$and where $M_{n}(n \in \mathbb{N})$ are distinct monomials. Then $\left(\mathcal{A}_{S},\|\cdot\|_{1}\right)$ is a commutative Banach algebra. Set $P_{\alpha}=\mathcal{A}_{S} \cap Q_{\alpha}$. Then $\left(P_{\alpha}: \alpha \in S\right)$ is an intersection non-redundant pseudo-finite family of prime ideals in $\mathcal{A}_{S}$.

We shall see further examples of commutative Banach algebras with or without an intersection non-redundant pseudo-finite sequence of prime ideals in $\$ \$ 8,9$, 10,

Lemma 3.9. Let $A$ and $\mathcal{A}$ be commutative algebras such that $\mathcal{A}$ contains $A$ as an ideal. Let $\left(P_{\alpha}: \alpha \in S\right)$ be an intersection non-redundant pseudo-finite family of prime ideals in $A$. Suppose that $\bigcup_{\alpha \in S} P_{\alpha} \neq A$. Then $\mathcal{A}$ also has an intersection non-redundant pseudo-finite family $\left(Q_{\alpha}: \alpha \in S\right)$ of prime ideals.

Proof. For each $\alpha \in S$, define

$$
Q_{\alpha}=\left\{a \in \mathcal{A}: a A \subset P_{\alpha}\right\} .
$$

It is standard that $Q_{\alpha}$ is a prime ideal in $\mathcal{A}$ and $Q_{\alpha} \cap A=P_{\alpha}$ (see [6, 1.3.53]).

Let $u \in A$ be such that $u \notin P_{\alpha}(\alpha \in S)$. We see that $Q_{\alpha}=\left\{a \in \mathcal{A}: a u \in P_{\alpha}\right\}$. The pseudo-finiteness of $\left(Q_{\alpha}: \alpha \in S\right)$ then follows. It is easily seen that this family is intersection non-redundant.

For the converse of the above lemma, we have the following.

Lemma 3.10. Let $A$ be a commutative algebra, and let I be an ideal in A. Suppose that each intersection non-redundant pseudo-finite family of prime ideals in each of the algebras $A / I$ and $I$ is finite. Then $A$ itself does not contain an intersection non-redundant pseudo-finite sequence of prime ideals.

Proof. Assume that there exists an intersection non-redundant pseudo-finite sequence $\left(P_{n}\right)$ of prime ideals in $A$. Letting $E$ be the subset of $\mathbb{N}$ consists of the numbers $n$ for which $I \not \subset P_{n}$. Then, for each $n \in E$, we have (again, by [6, 1.3.53])

$$
P_{n}=\left\{a \in A: a I \subset P_{n} \cap I\right\} .
$$

So we see that $\left\{P_{n} \cap I: n \in E\right\}$ is an intersection non-redundant pseudo-finite family of prime ideals in $I$. Thus $E$ must be finite.

On the other hand, it is readily seen that $\left\{P_{n} / I: n \in \mathbb{N} \backslash E\right\}$ is an intersection non-redundant pseudo-finite family of prime ideals in $A / I$. So, again, by the hypothesis, $\mathbb{N} \backslash E$ must be finite, a contradiction. Hence the result holds.

Definition 3.11. Let $A$ be a commutative algebra. Let $I$ be an ideal in $A$, and let $\left(f_{n}\right)$ be a sequence of elements in $A$. Then $I$ is extendible with respect to $\left(f_{n}\right)$ if both the following conditions hold:

(a) $f_{n}^{k} \notin I(n, k \in \mathbb{N})$, and $f_{m} f_{n} \in I(m \neq n \in \mathbb{N})$;

(b) for each $g \in A$, if $g f_{n_{0}}^{k_{0}} \in I$ for some $n_{0}, k_{0} \in \mathbb{N}$, then $g f_{n} \in I$ for all except finitely many $n \in \mathbb{N}$. 
Note that, if $A$ has an intersection non-redundant pseudo-finite sequence of prime ideals $\left(P_{n}\right)$, then, by setting $I=\bigcap_{n=1}^{\infty} P_{n}$ and choosing, for each $n \in \mathbb{N}$, an element $f_{n} \in \bigcap_{i \neq n} P_{i} \backslash P_{n}$, the ideal $I$ is extendible with respect to $\left(f_{n}\right)$. Conversely, we have the following general construction; this construction is crucial in our proof of the main result in $₫ 9$.

Proposition 3.12. Let $A$ be a commutative algebra. Suppose that there exist an ideal $I$ and a sequence $\left(f_{n}\right)$ in $A$ such that $I$ is extendible with respect to $\left(f_{n}\right)$. Then there exists a pseudo-finite sequence of prime ideals $\left(P_{n}\right)$ such that $f_{n} \in \bigcap_{i \neq n} P_{i} \backslash P_{n}$ for each $n \in \mathbb{N}$.

Proof. We see that the union of a chain of ideals, each of which contains $I$ and is extendible with respect to $\left(f_{n}\right)$, is also extendible with respect to $\left(f_{n}\right)$. By Zorn's lemma, we can choose a maximal one among those ideals, and call it $J$. It is easily seen that the prime radical of $J$ is again extendible with respect to $\left(f_{n}\right)$. Thus, by the maximality of $J$, we must have $\sqrt{J}=J$, and so $J$ is semiprime.

For each $n$, set $J_{n}=J: f_{n}$, and set $P=\bigcup_{n=1}^{\infty} J_{n}$. By the extensibility of $J$, we see that whenever $f \in P$ then $f \in J_{n}$ for all except finitely many $n \in \mathbb{N}$. Thus, in particular, the set $P$ is actually an ideal.

Claim 1: for each $f \in A \backslash P$, we have $J: f=J$. Indeed, since $f \notin P$ and since $J$ is semiprime, we see that $f_{n}^{k} \notin J: f(n, k \in \mathbb{N})$. It then follows easily that $J: f$ is extendible with respect to $\left(f_{n}\right)$. This and the maximality of $J$ imply the claim.

Claim 2: $P$ is either prime in $A$ or is $A$ itself. We have to prove that, whenever $f, g \in A$ are such that $f g \in P$, but $f \notin P$, then $g \in P$. Indeed, let $n_{0} \in \mathbb{N}$ be such that $f_{n_{0}} f g \in J$. Then $f_{n_{0}} g \in J: f$. The first claim then implies that $f_{n_{0}} g \in J$, and so $g \in J_{n_{0}}$.

Now, for each $n \in \mathbb{N}$, define

$$
S_{n}=\left\{f_{n}^{k}: k \in \mathbb{N}\right\} \cup\left\{f_{n}^{k} f: k \in \mathbb{N} \text { and } f \in A \backslash P\right\} .
$$

Then, by Claim 2, the set $S_{n}$ is closed under multiplication. We claim that $S_{n} \cap J_{n}=$ $\emptyset$. Indeed, fix $k \in \mathbb{N}$ and $f \in A \backslash P$. Then, since $f_{n}^{k+1} \notin J$, we see that $f_{n}^{k} \notin J_{n}$, and by Claim 1 , we see that $f_{n}^{k+1} f \notin J$, and so $f_{n}^{k} f \notin J_{n}$.

Since $S_{n}$ is closed under multiplication and $S_{n} \cap J_{n}=\emptyset$, there exists a prime ideal $P_{n}$ containing $J_{n}$ such that $S_{n} \cap P_{n}=\emptyset(n \in \mathbb{N})$. Then $J_{n} \subset P_{n} \subset P$ and $f_{n} \notin P_{n}(n \in \mathbb{N})$. The result then follows. (Indeed, it follows from the maximality of $J$ that $P_{n}=J_{n}$ for each $n$.)

\section{General necessary Condition}

In this section, we shall consider a homomorphism $\theta$ from a commutative Banach algebra $A$ into a Banach algebra $B$. To simplify the notations, set $\mathfrak{S}=\mathfrak{S}(\theta)$ and $\mathcal{I}=\mathcal{I}(\theta)$.

Let $\mathfrak{P}$ be the collection of all prime ideals in $A$ of the form $\mathcal{I}$ : $a$ for some $a \in A$. Note that $P \supset \mathcal{I}$ for each $P \in \mathfrak{P}$. The following lemma is a modification of the commutative prime kernel theorem due to Sinclair (see [6, Theorem 5.3.15] or [24, Theorem 11.4]); the proof is similar to that of the theorem, and is included here for the sake of completeness.

Lemma 4.1. Let $a_{0} \in A \backslash \sqrt{\mathcal{I}}$. Then there exists a prime ideal $P \in \mathfrak{P}$ such that $a_{0} \notin P$. In particular, $\sqrt{\mathcal{I}}$ is the intersection of prime ideals belonging to $\mathfrak{P}$. 
Proof. By the stability lemma, there exists $N \in \mathbb{N}$ such that

$$
\overline{\theta\left(a_{0}^{n}\right) \mathfrak{S}}=\overline{\theta\left(a_{0}^{N}\right) \mathfrak{S}} \quad(n \geq N) .
$$

Note that $a_{0}^{N} a_{0} \notin \mathcal{I}$. We claim that there exists $a_{1} \in A$ such that $a_{0}^{N} a_{1} \notin \mathcal{I}$ and such that, for each $a \in A$, either $\overline{\theta\left(a_{0}^{N} a_{1} a\right) \mathfrak{S}}=\overline{\theta\left(a_{0}^{N} a_{1}\right) \mathfrak{S}}$ or $a_{0}^{N} a_{1} a \in \mathcal{I}$. Indeed, assume the contrary. Then we can choose by induction a sequence $\left(a_{n}\right)$ in $A$ so that, for $n \in \mathbb{N}$, we have

$$
\overline{\theta\left(a_{0}^{N} a_{1}\right) \mathfrak{S}} \supsetneq \cdots \supsetneq \overline{\theta\left(a_{0}^{N} a_{1} \cdots a_{n-1}\right) \mathfrak{S}} \supsetneq \overline{\theta\left(a_{0}^{N} a_{1} \cdots a_{n}\right) \mathfrak{S}},
$$

contradicting the stability lemma. Thus the claim holds.

Define

$$
P=\mathcal{I}: a_{0}^{N} a_{1}=\left\{a \in A: \theta\left(a_{0}^{N} a_{1} a\right) \mathfrak{S}=\{0\}\right\} .
$$

We have $a_{0} \notin P$, for otherwise we would have $a_{0}^{N+1} a_{1} \in \mathcal{I}$, and this implies that $a_{0}^{N} a_{1} \in \mathcal{I}$ by equation (4.1), a contradiction of the fact that $a_{0}^{N} a_{1} \notin \mathcal{I}$. We now show that $P$ is a prime ideal. Let $a, b \in A$ with $a b \in P$, but $b \notin P$. Then

$$
0=\overline{\theta\left(a_{0}^{N} a_{1} a b\right) \mathfrak{S}} \supset \theta(a) \overline{\theta\left(a_{0}^{N} a_{1} b\right) \mathfrak{S}}=\theta(a) \overline{\theta\left(a_{0}^{N} a_{1}\right) \mathfrak{S}} \supset \theta\left(a_{0}^{N} a_{1} a\right) \mathfrak{S},
$$

and so $a \in P$ as required.

Definition 4.2. A collection $\mathcal{G}$ of subsets of a given set is said to be $F I$ if the set $\bigcap\{E: E \in \mathcal{G}\}$ can be represented as an intersection of a finite sub-collection of $\mathcal{G}$. We say that a collection $\mathcal{G}$ is $N F I$ if it has a sub-collection which is not FI.

So being not NFI means that all its sub-collections are FI.

Lemma 4.3. Suppose that $\mathfrak{P}$ is NFI. Then there exists $\mathcal{G} \subset \mathfrak{P}$ satisfying the following conditions:

(i) $\mathcal{G}$ is not FI;

(ii) for each $a \in A$, the set $\{P \in \mathcal{G}: a \notin P\}$ is either $\mathcal{G}$ itself or is not NFI.

Proof. For each $a \in A \cup\left\{\mathbf{e}_{A}\right\}$, let $\mathfrak{P}_{a}$ be the set of prime ideals of the form $\mathcal{I}$ : $a b$ for some $b \in A$. We claim that there exists $a_{0} \in A \cup\left\{\mathbf{e}_{A}\right\}$ such that $\mathfrak{P}_{a_{0}}$ is NFI and such that, for each $a \in A$, either $\mathfrak{P}_{a_{0} a}$ is not NFI or $\mathcal{I}: a_{0} a=\mathcal{I}: a_{0}$.

Assume the contrary. Then, since $\mathfrak{P}_{\mathbf{e}_{A}}$ is NFI, by induction, there exists a sequence $\left(a_{n}\right) \subset A$ such that $\mathfrak{P}_{a_{1} \cdots a_{n}}$ is NFI and such that

$$
\mathcal{I}: a_{1} \cdots a_{n} \subsetneq \mathcal{I}: a_{1} \cdots a_{n+1} \quad(n \in \mathbb{N}) .
$$

For each $n \in \mathbb{N}$, take $b_{n} \in \mathcal{I}: a_{1} \cdots a_{n+1} \backslash \mathcal{I}: a_{1} \cdots a_{n}$. By the stability lemma, there exists $N \in \mathbb{N}$ such that

$$
\overline{\theta\left(a_{1} \cdots a_{n}\right) \mathfrak{S}}=\overline{\theta\left(a_{1} \cdots a_{n+1}\right) \mathfrak{S}} \quad(n \geq N) .
$$

But then

$$
\theta\left(b_{n}\right) \overline{\theta\left(a_{1} \cdots a_{n}\right) \mathfrak{S}}=\theta\left(b_{n}\right) \overline{\theta\left(a_{1} \cdots a_{n+1}\right) \mathfrak{S}}=0 \quad(n \geq N),
$$

implying that $b_{n} a_{1} \cdots a_{n} \in \mathcal{I}(n \geq N)$. But this contradicts the choice of $\left(b_{n}\right)$. Hence the claim holds.

Since $\mathfrak{P}_{a_{0}}$ is NFI, there is a sub-collection $\mathcal{G}$ of $\mathfrak{P}_{a_{0}}$ which is not FI. Suppose that $a \in A$ and that $\mathcal{G}^{\prime}=\{P \in \mathcal{G}: a \notin P\}$ is NFI. Then, for each $P \in \mathcal{G}^{\prime}$, because $a \notin P$ we have $P: a=P$. Thus $\mathcal{G}^{\prime} \subset \mathfrak{P}_{a_{0} a}$, and hence $\mathfrak{P}_{a_{0} a}$ is NFI. Therefore, by 
the claim, we must have $\mathcal{I}: a_{0} a=\mathcal{I}: a_{0}$. We now show that $\mathcal{G}^{\prime}=\mathcal{G}$. Assume towards a contradiction that $\mathcal{G}^{\prime} \neq \mathcal{G}$, and let $P \in \mathcal{G} \backslash \mathcal{G}^{\prime}$, say $P=\mathcal{I}: a_{0} a_{1}$ for some $a_{1} \in A$. Then, since $a \in P$ we have $a_{1} \in \mathcal{I}: a_{0} a=\mathcal{I}: a_{0}$, so that $a_{0} a_{1} \in \mathcal{I}$. This implies that $P=A$, a contradiction. This proves that (ii) holds.

Lemma 4.4. Suppose that $\mathfrak{P}$ is NFI. Then there exists a sequence $\left(P_{n}\right) \subset \mathfrak{P}$ which is pseudo-finite and intersection non-redundant.

Proof. Let $\mathcal{G}$ be the sub-collection of $\mathfrak{P}$ as specified in Lemma 4.3 We claim that, in the partially ordered set $(\mathcal{G}, \subset)$, every descending chain must be eventually constant.

Assume the contrary. Then there exists a sequence $\left(Q_{n}\right)$ in $\mathcal{G}$ such that

$$
Q_{n} \supsetneq Q_{n+1} \quad(n \in \mathbb{N}) .
$$

Choose an element $a \in Q_{1} \backslash Q_{2}$. Then $\mathcal{G}^{\prime}:=\{P \in \mathcal{G}: a \notin P\}$ is NFI (since $\mathcal{G}^{\prime}$ contains $\left\{Q_{n}: n>1\right\}$, which is not FI), but $\mathcal{G}^{\prime} \neq \mathcal{G}$ (because $Q_{1} \notin \mathcal{G}^{\prime}$ ). But this contradicts Lemma 4.3 (ii). Thus, in $(\mathcal{G}, \subset)$, every element must contain a minimal element, and, since $\mathcal{G}$ is not FI, it must have infinitely many minimal elements.

Let $\left(P_{n}: n \in \mathbb{N}\right)$ be a sequence of distinct minimal elements of $(\mathcal{G}, \subset)$. Then obviously we have $P_{m} \not \subset P_{n}$ for each $m \neq n$ in $\mathbb{N}$.

Let $a \in \bigcup_{n=1}^{\infty} P_{n}$. By Lemma 4.3(ii), the set $\{P \in \mathcal{G}: a \notin P\}$ is not NFI, and so the set $\left\{P_{n}: a \notin P_{n}\right\}$ is FI. Let $n_{1}, \ldots, n_{k} \in \mathbb{N}$ be the indices such that

$$
\bigcap_{i=1}^{k} P_{n_{i}}=\bigcap\left\{P_{n}: a \notin P_{n}\right\} .
$$

For each $n \in \mathbb{N}$ with $a \notin P_{n}$, we have $\bigcap_{i=1}^{k} P_{n_{i}} \subset P_{n}$, and, since $P_{n}$ is a prime ideal, there exists $i \in\{1, \ldots, k\}$ such that $P_{n_{i}} \subset P_{n}$. Hence $n=n_{i}$. Thus

$$
\left\{n \in \mathbb{N}: a \notin P_{n}\right\}=\left\{n_{1}, \ldots, n_{k}\right\},
$$

and so $\left(P_{n}\right)$ is pseudo-finite. The rest then follows from Lemma 3.4 .

Now we can state the main theorem of this section.

Theorem 4.5. Let $\theta$ be a homomorphism from a commutative Banach algebra $A$ into a Banach algebra $B$ with continuity ideal $\mathcal{I}(\theta)=\mathcal{I}$. Then $\sqrt{\mathcal{I}(\theta)}$ is the intersection of the prime ideals of the form $\mathcal{I}$ : a for some $a \in A$.

Suppose further that $\sqrt{\mathcal{I}(\theta)}$ is not a finite intersection of prime ideals of the above form. Then there exist non-closed prime ideals $P_{n}=\mathcal{I}: a_{n}$, where $a_{n} \in A$ for each $n \in \mathbb{N}$, such that the sequence $\left(P_{n}: n \in \mathbb{N}\right)$ is pseudo-finite and intersection non-redundant, i.e., such that the following conditions hold:

(a) if a $\in \bigcup_{i=1}^{\infty} P_{i}$, then $a \in P_{n}$ for all but finitely many $n \in \mathbb{N}$;

(b) $\bigcap_{i \neq n} P_{i} \not \subset P_{n}(n \in \mathbb{N})$.

Proof. This follows in turn from Lemmas 4.1, 4.4, 3.6, and 3.4,

Lemma 4.6. Let I be a closed ideal in a commutative Banach algebra A such that $\sqrt{I}$ is also closed. Then there exists $k \in \mathbb{N}$ such that

$$
\sqrt{I}=\left\{a \in A: a^{k} \in I\right\} .
$$

Proof. This is an application of Baire's category theorem. 
Corollary 4.7. Let $\theta$ be an epimorphism from a commutative Banach algebra $A$ onto a Banach algebra $B$. Then:

(i) $\sqrt{\mathcal{I}(\theta)}$ is a finite intersection of prime ideals;

(ii) there is a number $k \in \mathbb{N}$ such that $\sqrt{\mathcal{I}(\theta)}=\left\{a \in A: a^{k} \in \mathcal{I}(\theta)\right\}$.

Proof. (i) Assume towards a contradiction that (i) is false. Let $P_{n}=\mathcal{I}: a_{n}(n \in \mathbb{N})$ be the prime ideals that were specified in Theorem 4.5. For $n \in \mathbb{N}$, set

$$
Q_{n}=\left\{b \in B: b \theta\left(a_{n}\right) \mathfrak{S}=\{0\}\right\} .
$$

Then $Q_{n}$ is closed in $B$. We see that $P_{n}=\theta^{-1}\left(Q_{n}\right)$ and that $Q_{n}=\theta\left(P_{n}\right)$, and so $Q_{n}$ is a prime ideal in $B$. It is easily seen that $\left(Q_{n}: n \in \mathbb{N}\right)$ is pseudo-finite and intersection non-redundant, contradicting Lemma 3.6. Thus (i) holds.

(ii) Define $\mathcal{J}=\{b \in B: b \mathfrak{S}=\{0\}\}$. Then $\mathcal{J}$ is a closed ideal in $B$. We see that $\mathcal{I}=\theta^{-1}(\mathcal{J})$ and $\mathcal{J}=\theta(\mathcal{I})$. This and Lemma 4.1 imply that $\sqrt{\mathcal{J}}$ is the intersection of the ideals of the form $\mathcal{J}: b$ for $b \in B$. So $\sqrt{\mathcal{J}}$ is closed in $B$, since each $\mathcal{J}: b$ is closed. We can then apply Lemma 4.6.

Remark. In the case where $B$ is also a semiprime algebra, we see that $\mathcal{I}(\theta)$ is either $A$ or a semiprime ideal, and so $\mathcal{I}(\theta)=\sqrt{\mathcal{I}(\theta)}$. Hence $\mathcal{I}(\theta)$ is a finite intersection of prime ideals. Note, however, that this does not help in answering the (still open) question of whether or not there exists a discontinuous epimorphism from a Banach algebra onto a commutative semiprime Banach algebra; see 2 for some partial results on this question.

We turn to another application. Let $D: A \rightarrow M$ be a derivation from a commutative Banach algebra $A$ into a Banach $A$-bimodule $M$. We know that the continuity ideal $\mathcal{I}(D)$ of $D$ is the continuity ideal of a homomorphism from $A$ into some Banach algebra and that $\mathcal{I}(D)$ is closed (see $\oint_{2}$ ). So, for each $a \in A$, the ideal $\mathcal{I}(D): a$ is closed. Thus, we have the following corollary.

Corollary 4.8. Let $D$ be a derivation from a commutative Banach algebra $A$ into a Banach A-bimodule $M$. Then:

(i) $\sqrt{\mathcal{I}(D)}$ is a finite intersection of closed prime ideals;

(ii) there is a number $k \in \mathbb{N}$ such that $\sqrt{\mathcal{I}(D)}=\left\{a \in A: a^{k} \in \mathcal{I}(D)\right\}$.

\section{Continuity ideals Which are nOt intersections of PRime ideals}

From the previous section, we may ask whether the continuity ideal of a homomorphism is necessarily either a semiprime ideal or the whole algebra itself (see also Theorem 1.1(iv)). In this section, counter-examples to this possibility will be presented. It will be shown that although the continuity ideal of an epimorphism or a derivation is close to being an intersection of prime ideals, as shown by Corollaries 4.7(ii) and 4.8(ii), this is not always the case. The epimorphism we shall construct is in fact an automorphism. By the remark after Corollary 4.7, the domain of this automorphism cannot be a semiprime algebra. However, it is possible to construct a desired derivation from a semisimple algebra. It can even be arranged so that the derivation maps into the dual of the algebra.

Denote by $\mathbb{D}$ the open unit disk $\{z \in \mathbb{C}:|z|<1\}$. Define $A\left(\overline{\mathbb{D}}^{n}\right)$ to be the Banach algebra of complex functions of $n$ complex variables continuous on $\overline{\mathbb{D}}^{n}$ and holomorphic in $\mathbb{D}^{n}$, with the uniform norm; we are interested in the cases where $n=1,2$. 
Theorem 5.1. Let $\mathcal{A}=A\left(\overline{\mathbb{D}}^{2}\right)$. Then there exists a derivation $\mathcal{D}$ from $\mathcal{A}$ into its dual $\mathcal{A}^{\prime}$ such that $\mathcal{I}(\mathcal{D})$ is not an intersection of prime ideals in $\mathcal{A}$.

Proof. We proceed in two steps.

Step 1: Let $A$ be the disk algebra $A(\overline{\mathbb{D}})$. Denote by $\mathbf{1}$ the function $\mathbf{1}(z) \equiv 1$. By [6] 5.6.81, 5.2.17], there exists a discontinuous derivation $D: A \rightarrow A^{\prime}$. Then we must have $\mathbf{1} \notin \mathcal{I}(D)$.

Next, we consider $A_{1}=A \oplus A$ with $\ell^{1}$-norm, with pointwise addition, and with product defined by

$$
\left(f_{1} \oplus f_{2}\right)\left(g_{1} \oplus g_{2}\right)=f_{1} g_{1} \oplus\left(f_{1} g_{2}+f_{2} g_{1}\right) \quad\left(f_{1}, f_{2}, g_{1}, g_{2} \in A\right) .
$$

Then $A_{1}$ is a commutative Banach algebra. Then, we define left and right actions of $A_{1}$ on $A^{\prime} \oplus A^{\prime}$ as follows:

$$
\left(\lambda_{1} \oplus \lambda_{2}\right) \cdot\left(f_{1} \oplus f_{2}\right)=\left(f_{1} \oplus f_{2}\right) \cdot\left(\lambda_{1} \oplus \lambda_{2}\right)=\left(f_{1} \cdot \lambda_{1}+f_{2} \cdot \lambda_{2}\right) \oplus\left(f_{1} \cdot \lambda_{2}\right),
$$

for each $f_{1} \oplus f_{2} \in A_{1}$ and each $\lambda_{1} \oplus \lambda_{2} \in A^{\prime} \oplus A^{\prime}$. We can check that $A^{\prime} \oplus A^{\prime}$ with pointwise addition, with the above $A_{1}$ actions, and with maximum norm is a Banach $A_{1}$-bimodule. In fact, as Banach $A_{1}$-bimodules, $A^{\prime} \oplus A^{\prime}$ is isometrically isomorphic to $(A \oplus A)^{\prime}$ with the following pairing:

$$
\left\langle\lambda_{1} \oplus \lambda_{2}, f_{1} \oplus f_{2}\right\rangle=\lambda_{1}\left(f_{1}\right)+\lambda_{2}\left(f_{2}\right) \quad\left(\lambda_{1}, \lambda_{2} \in A^{\prime}, f_{1}, f_{2} \in A\right) .
$$

Define a linear map $D_{1}: A_{1} \rightarrow A^{\prime} \oplus A^{\prime}$ by

$$
D_{1}\left(f_{1} \oplus f_{2}\right)=D f_{2} \oplus D f_{1} \quad\left(f_{1}, f_{2} \in A\right) .
$$

Then it is easy to verify that $D_{1}$ is a derivation. We now compute $\mathcal{I}\left(D_{1}\right)$. By definition, $f_{1} \oplus f_{2} \in \mathcal{I}\left(D_{1}\right)$ if and only if the map

$$
g_{1} \oplus g_{2} \mapsto\left(f_{1} \cdot D g_{2}+f_{2} \cdot D g_{1}\right) \oplus\left(f_{1} \cdot D g_{1}\right), \quad A_{1} \rightarrow A^{\prime} \oplus A^{\prime},
$$

is continuous; that is, if and only if $f_{1} \oplus f_{2} \in \mathcal{I}(D) \oplus \mathcal{I}(D)$. In particular, we have $\{0\} \oplus A \not \subset \mathcal{I}\left(D_{1}\right)$, since $\mathbf{1} \notin \mathcal{I}(D)$. But $(\{0\} \oplus A)^{2}=\{0\}$, and so $\mathcal{I}\left(D_{1}\right)$ is not a semiprime ideal.

Step 2: We now consider $D_{1}$ as a derivation from $A_{1}$ into $A_{1}^{\prime}$. For each function $f\left(Z_{1}, Z_{2}\right) \in \mathcal{A}=A\left(\overline{\mathbb{D}}^{2}\right)$, denote by $\partial_{2} f$ the partial derivative of $f$ with respect to the second variable $Z_{2}$. Define a map

$$
\Phi: f\left(Z_{1}, Z_{2}\right) \mapsto f\left(Z_{1}, 0\right) \oplus\left(\partial_{2} f\right)\left(Z_{1}, 0\right), \quad \mathcal{A} \rightarrow A_{1} .
$$

Then $\Phi$ is an algebra epimorphism from $\mathcal{A}$ onto $A_{1}$, and, by Cauchy's estimation theorem, $\Phi$ is bounded. Consider $A_{1}$ as a Banach $\mathcal{A}$-bimodule with module multiplications defined, for each $f \in \mathcal{A}$ and each $\left(g_{1}, g_{2}\right) \in A_{1}$, as

$$
f \cdot\left(g_{1} \oplus g_{2}\right)=\left(g_{1} \oplus g_{2}\right) \cdot f=\Phi(f) \cdot\left(g_{1} \oplus g_{2}\right)=\left(g_{1} \oplus g_{2}\right) \cdot \Phi(f) .
$$

Then $\Phi$ is also an $\mathcal{A}$-bimodule epimorphism, and so the adjoint map $\Phi^{\prime}: A_{1}^{\prime} \rightarrow \mathcal{A}^{\prime}$ is a Banach $\mathcal{A}$-bimodule embedding (onto a closed submodule of $\mathcal{A}^{\prime}$ ).

Finally, define a map $\mathcal{D}: \mathcal{A} \rightarrow \mathcal{A}^{\prime}$ by setting $\mathcal{D}=\Phi^{\prime} \circ D_{1} \circ \Phi$. Then $\mathcal{D}$ is a derivation from $\mathcal{A}$ into $\mathcal{A}^{\prime}$. We can check that $\mathcal{I}(\mathcal{D})=\Phi^{-1}\left[\mathcal{I}\left(D_{1}\right)\right]$, which is, by Step 1 , neither a semiprime ideal in $\mathcal{A}$ nor $\mathcal{A}$ itself.

This complete the proof.

Thus, there is a homomorphism from the semisimple commutative Banach algebra $A\left(\overline{\mathbb{D}}^{2}\right)$ into another Banach algebra whose continuity ideal $\mathcal{I}$ is not equal to its prime radical $\sqrt{\mathcal{I}}$. We can even find an automorphism with this property; 
however, the cost of this (using our construction) is that the underlying algebra is "unnatural".

Corollary 5.2. There exists an automorphism $\theta$ on a commutative Banach algebra such that $\mathcal{I}(\theta)$ is not an intersection of prime ideals.

Proof. Let $D: A \rightarrow M$ be any derivation from a commutative Banach algebra $A$ into a commutative Banach $A$-bimodule $M$ such that $\mathcal{I}(D)$ is a proper ideal but not a semiprime ideal in $A$. Consider $A \oplus M$ as a commutative Banach algebra as before. Define a map $\theta: A \oplus M \rightarrow A \oplus M$ by

$$
\theta(a \oplus x)=a \oplus(D a+x) \quad(a \in A, x \in M) .
$$

It is easily checked that $\theta$ is an automorphism of $A \oplus M$. It follows routinely that $\mathcal{I}(\theta)=\mathcal{I}(D) \oplus M$, which is neither a semiprime ideal in $A \oplus M$ nor $A \oplus M$ itself.

\section{MAPpings IntO ULTRAPOWERS}

First, we need some definitions. Let $\kappa$ be an infinite cardinal. Then $\mathbb{C}^{\kappa}$ with pointwise algebraic operations is an algebra. Let $\mathcal{U}$ be a free ultrafilter on $\kappa$. Define $M_{\mathcal{U}}$ to be the set of all elements $f \in \mathbb{C}^{\kappa}$ such that $\{\sigma \in \kappa: f(\sigma)=0\} \in \mathcal{U}$. Then $M_{\mathcal{U}}$ is a maximal ideal. The field $\mathbb{C}^{\kappa} / M_{\mathcal{U}}$ is called an ultrapower, and is denoted by $\mathbb{C}^{\kappa} / \mathcal{U}$. An element $f+M_{\mathcal{U}} \in \mathbb{C}^{\kappa} / \mathcal{U}$ is an infinitesimal if

$$
\{\sigma \in \kappa:|f(\sigma)|<\varepsilon\} \in \mathcal{U}
$$

for each $\varepsilon>0$. The subalgebra of infinitesimals of $\mathbb{C}^{\kappa} / \mathcal{U}$ is denoted by $\left(\mathbb{C}^{\kappa} / \mathcal{U}\right)^{\circ}$.

Now let $\mathcal{V}$ be a free ultrafilter on $\mathbb{N}$. Recall that $c_{0}$ is the algebra of the sequences of complex numbers converging to 0 (with pointwise operations). Let $P_{\mathcal{V}}$ be the set of all sequences $\left(x_{n}\right) \in c_{0}$ such that $\left\{n \in \mathbb{N}: x_{n}=0\right\} \in \mathcal{V}$. Then $P_{\mathcal{V}}$ is a prime ideal in $c_{0}$, and $c_{0} / P_{\mathcal{V}}$ is an integral domain which can be naturally identified with a subalgebra of $\left(\mathbb{C}^{\mathbb{N}} / \mathcal{V}\right)^{\circ}$. We denote $c_{0} / P_{\mathcal{V}}$ by $c_{0} / \mathcal{V}$. Thus each $c_{0} / \mathcal{V}$ is indeed a quotient of $\mathcal{C}_{0}(\mathbb{N})$ by one of its minimal prime ideals.

In their approach to the construction in [13] of an embedding of an arbitrary nonunital integral domain of cardinality $\mathfrak{c}$ into a radical Banach algebra, the authors of [9] used a special case that $c_{0} / \mathcal{V}$ can be embedded into some radical Banach algebra for any ultrafilter $\mathcal{V}$ on $\mathbb{N}$ (this "classical" case had been constructed by Dales and Esterle in their solution to the conjecture of Kaplansky) - this part requires $\mathrm{CH}$, together with the following theorem (which is [9, Theorem 5.25], and does not require the Continuum Hypothesis).

Theorem 6.1. Let $A$ be a non-unital integral domain of cardinality $\mathfrak{c}$. Then there exists an embedding of $A$ into $c_{0} / \mathcal{V}$ for some free ultrafilter $\mathcal{V}$ on $\mathbb{N}$.

Our aim in this section is to extend the above result; the construction of embeddings into radical Banach algebras will be extended in the next section. However, it should be noted that much difficulty in both of these extensions is actually hidden behind the results that they extend.

We shall need some basic complex algebraic-geometry results to prove the main theorem of this section. Our references for algebraic geometry will be [20] and 25]. Some conventions: all our varieties will be algebraic and the only topology considered on complex spaces is the Euclidean topology. For a set $S \subset \mathbb{C}\left[Z_{1}, Z_{2}, \ldots, Z_{n}\right]$, denote by $\mathcal{V}(S)$ the variety (i.e., common zero set) of $S$ in $\mathbb{C}^{n}$. For each prime ideal 
$Q$ in $\mathbb{C}\left[Z_{1}, \ldots, Z_{n}\right]$, the variety $\mathcal{V}(Q)$ is irreducible. We shall many times use the following proposition.

Proposition 6.2. Let $V$ be an irreducible variety of $\mathbb{C}^{n}$, and let $W$ be a proper subvariety of $V$. Then $V \backslash W$ is (relatively) open and dense in $V$.

Proof. Clearly, $V \backslash W$ is relatively open in $V$. The density follows from Theorem 2.11 in [20, Chapter IV].

Notation. For clarity, we shall use $X_{i}, Y_{j}$ for variables, $x_{i}, y_{j}$ for complex numbers, and $a_{i}, b_{j}$ for elements of an algebra. When there is no ambiguity, we shall use boldface characters to denote tuples of elements of the same type; for example, we set

$$
\boldsymbol{X}=\left(X_{1}, X_{2}, \ldots, X_{m}\right) \quad \text { or } \quad \boldsymbol{y}=\left(y_{1}, \ldots, y_{n}\right) .
$$

In the case where $\boldsymbol{X}=\left(X_{1}, \ldots, X_{m}\right)$, we also denote by $\mathbb{C}_{\boldsymbol{X}}$ the corresponding space $\mathbb{C}^{m}$.

Lemma 6.3. Let $m \in \mathbb{N}$ and $n \in \mathbb{Z}^{+}$, and let $Q$ be a prime ideal in $\mathbb{C}[\boldsymbol{X}, \boldsymbol{Y}]$, where $\boldsymbol{X}=\left(X_{1}, \ldots, X_{m}\right)$ and $\boldsymbol{Y}=\left(Y_{1}, \ldots, Y_{n}\right)$. Suppose that $X_{1}, \ldots, X_{m}$ are algebraically independent modulo $Q$. Let $V$ be the variety of $Q$. Let $\pi$ be the natural projection $\mathbb{C}_{\boldsymbol{X}, \boldsymbol{Y}} \rightarrow \mathbb{C}_{\boldsymbol{X}}$. Then there exists a dense open subset $U$ of $V$ such that $\pi: U \rightarrow \mathbb{C}_{\boldsymbol{X}}$ is an open map.

Proof. Let $\left\{Y_{j_{1}}, Y_{j_{2}}, \ldots, Y_{j_{k}}\right\}$ be a maximal subset of $\left\{Y_{1}, \ldots, Y_{n}\right\}$ such that

$$
\left\{X_{1}, \ldots, X_{m}, Y_{j_{1}}, \ldots, Y_{j_{k}}\right\}
$$

is algebraically independent modulo $Q$. By renaming $Y_{j_{t}}$ as $X_{m+t}(1 \leq t \leq k)$ and the remaining variables $Y_{j}$ as $Y_{1}, \ldots, Y_{n-k}$, we may suppose that the set $\left\{X_{1}, \ldots, X_{m}\right\}$ is indeed a transcendence basis for $\mathbb{C}[\boldsymbol{X}, \boldsymbol{Y}]$ modulo $Q$. If $n=0$, then $Q=\{0\}$, and so $U=V=\mathbb{C}_{\boldsymbol{X}}$ is the desired set. From now on, we shall suppose that $n \geq 1$. For each $1 \leq j \leq n$, we choose a polynomial

$$
p_{j}=q_{j, 0} Y_{j}^{k_{j}}+q_{j, 1} Y_{j}^{k_{j}-1}+\cdots+q_{j, k_{j}} \in Q
$$

with all $q_{j, t} \in \mathbb{C}\left[\boldsymbol{X}, Y_{1}, \ldots, Y_{j-1}\right]$ and $q_{j, 0} \notin Q$, and such that $p_{j}$ is a minimal polynomial of this kind (i.e., with smallest degree in $Y_{j}$ ).

Now, for $1 \leq j \leq n$, let $p \in Q \cap \mathbb{C}\left[\boldsymbol{X}, Y_{1}, \ldots, Y_{j}\right]$ be arbitrary. Using the division algorithm in $\mathbb{C}\left[\boldsymbol{X}, Y_{1}, \ldots, Y_{j-1}\right]\left[Y_{j}\right]$ and the minimality of $p_{j}$, we see that, for some $l \in \mathbb{N}$, when dividing $\left(q_{j, 0}\right)^{l} p$ by $p_{j}$ the remainder is a polynomial in $Y_{j}$ with coefficients in $Q \cap \mathbb{C}\left[\boldsymbol{X}, Y_{1}, \ldots, Y_{j-1}\right]$. Applying this inductively and using the fact that $Q \cap \mathbb{C}[\boldsymbol{X}]=\{0\}$, we obtain, for each $p \in Q$, after multiplying $p$ by some power of $q_{1,0} \cdots q_{n, 0}$, a polynomial in the ideal generated by $p_{1}, \ldots, p_{n}$. So, if $(\boldsymbol{x}, \boldsymbol{y})$ is not in $\mathcal{V}\left(q_{1,0} \cdots q_{n, 0}\right)$, then it is in $\mathcal{V}\left(p_{1}, \ldots, p_{n}\right)$ if and only if it is in $V$.

Also, by the minimality of each $p_{j}$, we have $\partial p_{j} / \partial Y_{j}$ is not in $Q(1 \leq j \leq n)$. So

$$
\mathcal{V}\left(q_{1,0} \cdots q_{n, 0} \frac{\partial p_{1}}{\partial Y_{1}} \cdots \frac{\partial p_{n}}{\partial Y_{n}}\right)
$$

is a variety which does not contain $V$. Thus, by setting

$$
U=V \backslash \mathcal{V}\left(q_{1,0} \cdots q_{n, 0} \frac{\partial p_{1}}{\partial Y_{1}} \cdots \frac{\partial p_{n}}{\partial Y_{n}}\right)
$$


we see, by Proposition 6.2, that $U$ is a dense open subset of $V$. Fix a point $\left(\boldsymbol{x}_{0}, \boldsymbol{y}_{0}\right) \in U$. Then the right $n \times n$ submatrix of the Jacobian

$$
\left(\begin{array}{ccc|ccc}
\frac{\partial p_{1}}{\partial X_{1}} & \cdots & \frac{\partial p_{1}}{\partial X_{m}} & \frac{\partial p_{1}}{\partial Y_{1}} & \cdots & 0 \\
\vdots & \ddots & \vdots & \vdots & \ddots & \vdots \\
\frac{\partial p_{n}}{\partial X_{1}} & \cdots & \frac{\partial p_{n}}{\partial X_{m}} & \cdots & \cdots & \frac{\partial p_{n}}{\partial Y_{n}}
\end{array}\right)
$$

of the map $\left(p_{1}, \ldots, p_{n}\right): \mathbb{C}_{\boldsymbol{X}, \boldsymbol{Y}} \rightarrow \mathbb{C}^{n}$ at $\left(\boldsymbol{x}_{0}, \boldsymbol{y}_{0}\right)$ is lower triangular, with non-zero diagonal entries. So this submatrix is invertible in an entire neighbourhood $\Delta$ (in $\left.\mathbb{C}_{\boldsymbol{X}, \boldsymbol{Y}}\right)$ of $\left(\boldsymbol{x}_{0}, \boldsymbol{y}_{0}\right)$. The result then follows from the implicit mapping theorem.

Lemma 6.4. Let $A$ be a non-unital commutative algebra. Let $P$ and $P_{r}$ be ideals in $A$ which are prime in $A^{\#}$ and such that $P \supset P_{r}(r \in \mathbb{N})$. Let $m, n \in \mathbb{Z}^{+}$with $m+n \geq 1$, and let $\boldsymbol{a}=\left(a_{1}, \ldots, a_{m}\right)$ and $\boldsymbol{b}=\left(b_{1}, \ldots, b_{n}\right)$ be finite sequences in $A$ such that $\left\{a_{1}, \ldots, a_{m}\right\}$ is algebraically independent modulo $P$. Let $\delta>0$. Then there exist finite sequences of complex numbers

$$
\left(\boldsymbol{x}, \boldsymbol{y}^{(r)}\right)=\left(x_{1}, \ldots, x_{m}, y_{1}^{(r)}, \ldots, y_{n}^{(r)}\right) \quad(r \in \mathbb{N})
$$

satisfying all the following conditions:

(i) $p\left(\boldsymbol{x}, \boldsymbol{y}^{(r)}\right)=0$ for each $r \in \mathbb{N}$ and each $p \in \mathbb{C}[\boldsymbol{X}, \boldsymbol{Y}]$ with $p(\boldsymbol{a}, \boldsymbol{b}) \in P_{r}$;

(ii) $x_{i} \neq 0 \quad(1 \leq i \leq m)$;

(iii) for each $r \in \mathbb{N}$ and each $1 \leq j \leq n$, we have $y_{j}^{(r)} \neq 0$ whenever $b_{j} \notin P_{r}$;

(iv) $\left|x_{i}\right| \leq \delta$ and $\left|y_{j}^{(r)}\right| \leq \delta \quad(1 \leq i \leq m, 1 \leq j \leq n, r \in \mathbb{N})$.

Proof. Without loss of generality, we can assume that $a_{1}, \ldots, a_{m}$ are distinct. Define $Q=\{p \in \mathbb{C}[\boldsymbol{X}, \boldsymbol{Y}]: p(\boldsymbol{a}, \boldsymbol{b}) \in P\}$, and, for each $r \in \mathbb{N}$, define

$$
Q_{r}=\left\{p \in \mathbb{C}[\boldsymbol{X}, \boldsymbol{Y}]: p(\boldsymbol{a}, \boldsymbol{b}) \in P_{r}\right\} .
$$

Then $Q_{r} \subset Q$ are prime ideals in $\mathbb{C}[\boldsymbol{X}, \boldsymbol{Y}]$. We also see that $\left\{X_{1}, \ldots, X_{m}\right\}$ is algebraically independent modulo $Q$, and hence modulo $Q_{r}$ for each $r$. Let $V$ and $V_{r}$ be the varieties corresponding to $Q$ and $Q_{r}$, respectively. Then $V \subset V_{r}$. If $m=0$, then we set $U=V$ and $U_{r}=V_{r}\left(r \in \mathbb{N}\right.$ ); otherwise, let $U$ (respectively, $U_{r}$ ) be the dense open subset of $V$ (respectively, $V_{r}$ ) specified in Lemma 6.3. For each $r \in \mathbb{N}$, set

$$
W_{r}=\bigcup_{i=1}^{m}\left\{(\boldsymbol{x}, \boldsymbol{y}): x_{i}=0\right\} \cup \bigcup_{1 \leq j \leq n, b_{j} \notin P_{r}}\left\{(\boldsymbol{x}, \boldsymbol{y}): y_{j}=0\right\} .
$$

Then $W_{r}$ is a variety which does not contain $V_{r}$, and so $V_{r} \backslash W_{r}$ is also a dense open subset of $V_{r}$ because $V_{r}$ is irreducible. Therefore, $U_{r} \backslash W_{r}$ is again a dense open subset of $V_{r}$. Set

$$
\Delta=\left\{(\boldsymbol{x}, \boldsymbol{y}):\left|x_{i}\right|<\delta(1 \leq i \leq m) \text { and }\left|y_{j}\right|<\delta(1 \leq j \leq n)\right\} .
$$

Finally, set $U_{r}^{\prime}=\left(U_{r} \backslash W_{r}\right) \cap \Delta$ and $U^{\prime}=U \cap \Delta$. Note that the origin $\mathbf{0}$ is in $V$ and $V_{r}\left(r \in \mathbb{N}\right.$ ). So $U^{\prime}$ (respectively, $U_{r}^{\prime}$ ) is a non-empty open subset of $V$ (respectively, $\left.V_{r}\right)$.

Case 1: $m=0$. We have $\boldsymbol{x}=\emptyset$.

Case 2: $m \geq 1$. We have $\pi\left(U^{\prime}\right)$ and $\pi\left(U_{r}^{\prime}\right)(r \in \mathbb{N})$ are open in $\mathbb{C}_{\boldsymbol{X}}$ by Lemma 6.3. where $\pi: \mathbb{C}_{\boldsymbol{X}, \boldsymbol{Y}} \rightarrow \mathbb{C}_{\boldsymbol{X}}$ is the natural projection. Now, since $U_{r} \backslash W_{r}$ is dense in $V_{r}$ and $U^{\prime} \subset V \subset V_{r}$, we have $\pi\left(U_{r}^{\prime}\right) \cap \pi\left(U^{\prime}\right)$ is dense (and open) in $\pi\left(U^{\prime}\right)$. By the Baire category theorem, there exists $\boldsymbol{x} \in \bigcap_{r=1}^{\infty} \pi\left(U_{r}^{\prime}\right)$. 
In both cases, for each $r$, choose $\left(\boldsymbol{x}, \boldsymbol{y}^{(r)}\right) \in U_{r}^{\prime}$. These are the sequences we need.

We can now prove the following extension of [9, Theorem 24].

Theorem 6.5. Let $A$ be a commutative algebra. Let $\left(P_{\alpha}: \alpha \in S\right)$ be a pseudo-finite family of non-modular prime ideals in $A$ indexed by a non-empty set $S$. Then there exist a cardinal $\kappa$, a free ultrafilter $\mathcal{U}$ on $\kappa$, and, for each $\alpha \in S$, a homomorphism $\theta_{\alpha}: A \rightarrow\left(\mathbb{C}^{\kappa} / \mathcal{U}\right)^{\circ}$ such that:

(a) $\operatorname{ker} \theta_{\alpha}=P_{\alpha}(\alpha \in S)$, and

(b) the set $\left\{\theta_{\alpha}(a): \alpha \in S\right\}$ is finite for each $a \in A$.

Proof. Note that $A$ must be non-unital. Since each $P_{\alpha}$ is a non-modular prime ideal in $A$, it is a prime ideal in $A^{\#}$. If $S$ is finite, set $P=A$; otherwise, set $P=\bigcup_{\alpha \in S} P_{\alpha}$. In both cases, we have $P$ is a prime ideal in $A^{\#}$.

Let $\Gamma \subset A$ be a transcendence base for $A^{\#}$ modulo $P$. (Note that $\Gamma=\emptyset$ if $P=A$.) Then we have $\operatorname{alg} \Gamma \cap P=\{0\}$. Set $A_{0}=\operatorname{alg} \Gamma+P$. Then $A_{0}$ is a subalgebra of $A$. We see that each $a \in A$ satisfies a polynomial of the form $a_{0} X^{m}+a_{1} X^{m-1}+\cdots+a_{m}$, with $a_{0}, \ldots, a_{m-1} \in \operatorname{alg} \Gamma+\mathbb{C}, a_{0} \neq 0$, and $a_{m} \in A_{0}$.

Let $\kappa$ be the set of all tuples of the form $\left(\delta ; F ; a_{1}, \ldots, a_{m}\right)$, where $\delta>0, F$ is a finite subset of $S$, and $\left(a_{1}, \ldots, a_{m}\right)$ is a non-empty finite sequence in $A$. Define a partial order $\prec$ on $\kappa$ by setting

$$
\left(\delta ; F ; a_{1}, a_{2}, \ldots, a_{m}\right) \prec\left(\delta^{\prime} ; F^{\prime} ; a_{1}^{\prime}, a_{2}^{\prime}, \ldots, a_{m^{\prime}}^{\prime}\right)
$$

if $\delta>\delta^{\prime}, F \subset F^{\prime}$, and $\left(a_{1}, \ldots, a_{m}\right)$ is a subsequence of $\left(a_{1}^{\prime}, a_{2}^{\prime}, \ldots, a_{m^{\prime}}^{\prime}\right)$. Then $(\kappa, \prec)$ is a net. Fix an ultrafilter $\mathcal{U}$ on $\kappa$ majorizing this net.

By Lemma 6.4 for each $w=\left(\delta ; F ; a_{1}, a_{2}, \ldots, a_{m}\right) \in \kappa$, we can find sequences $\tau_{\alpha}(w)=\left(x_{1}^{(\alpha)}, x_{2}^{(\alpha)}, \ldots, x_{m}^{(\alpha)}\right) \in \mathbb{C}^{m}$ depending on $w$ (for $\alpha \in F$ ) such that:

(i) for each $\alpha \in F$ and each $p \in \mathbb{C}\left[X_{1}, \ldots, X_{m}\right]$ with $p\left(a_{1}, \ldots, a_{m}\right) \in P_{\alpha}$, we have $p\left(x_{1}^{(\alpha)}, \ldots, x_{m}^{(\alpha)}\right)=0$

(ii) for each $\alpha \in F$ and each $1 \leq k \leq m$ with $a_{k} \notin P_{\alpha}$, we have $x_{k}^{(\alpha)} \neq 0$;

(iii) $\left|x_{k}^{(\alpha)}\right| \leq \delta \quad(1 \leq k \leq m, \alpha \in F)$;

(iv) for each $1 \leq k \leq m$ with $a_{k} \in \Gamma$, we have $x_{k}^{(\alpha)}=x_{k}^{(\beta)}(\alpha, \beta \in F)$.

We then set $\tau_{\alpha}(w)=\mathbf{0} \in \mathbb{C}^{m}$ for each $\alpha \in S \backslash F$.

Define $\psi_{\alpha}: A \rightarrow \mathbb{C}^{\kappa}$ as follows: for each $a \in A$ and each

$$
w=\left(\delta ; F ; a_{1}, \ldots, a_{m}\right) \in \kappa
$$

such that $\alpha \in F$ and such that $a$ is in $w$, say $a=a_{k}$, then $\psi_{\alpha}(a)(w)=\tau_{\alpha}(w)(k)$; otherwise, $\psi_{\alpha}(a)(w)=0$. Note that $\psi_{\alpha}$ is well-defined, for, if $a_{j}=a_{k}$ and $\alpha \in F$, then $a_{j}-a_{k}=0 \in P_{\alpha}$, so that $\tau_{\alpha}(w)(j)=\tau_{\alpha}(w)(k)$ by (i). Define $\theta_{\alpha}(a)$ to be the equivalence class in $\mathbb{C}^{\kappa} / \mathcal{U}$ containing $\psi_{\alpha}(a)$.

Let $\alpha \in S$. By (i), the map $\theta_{\alpha}$ is an algebra homomorphism and $P_{\alpha} \subset \operatorname{ker} \theta_{\alpha}$; by combining this with (ii), we see that $\operatorname{ker} \theta_{\alpha}$ is exactly $P_{\alpha}$. By (iii), the image of $\theta_{\alpha}$ is contained in $\left(\mathbb{C}^{\kappa} / \mathcal{U}\right)^{\circ}$. The requirement (b) is trivially satisfied if $S$ is finite. So, for the rest of the proof, we shall assume that $S$ is infinite. By (iv), for each $a \in \operatorname{alg} \Gamma$, the family $\left(\theta_{\alpha}(a): \alpha \in S\right)$ is constant. Combining this with the pseudo-finiteness of $\left(P_{\alpha}\right)$, we see that, for each $a \in A_{0}=\operatorname{alg} \Gamma+\bigcup P_{\alpha}$, the set $\left\{\theta_{\alpha}(a): \alpha \in S\right\}$ is finite. The next lemma will complete the proof. 
Lemma 6.6. Let $A,\left(P_{\alpha}: \alpha \in S\right), \Gamma$, and $A_{0}$ be as in the previous theorem. Let $D$ be a non-unital integral domain. Suppose that we have homomorphisms $\theta_{\alpha}: A \rightarrow D$ such that $\operatorname{ker} \theta_{\alpha} \cap \operatorname{alg} \Gamma=\{0\} \quad(\alpha \in S)$, and such that, for each $a \in A_{0}$, the set $\left\{\theta_{\alpha}(a): \alpha \in S\right\}$ is finite. Then, for each $a \in A$, the set $\left\{\theta_{\alpha}(a): \alpha \in S\right\}$ is finite.

Proof. Obviously, $D^{\#}$ is also an integral domain. Let $a \in A$. Choose elements $a_{0}, \ldots, a_{m-1} \in \operatorname{alg} \Gamma, x_{0}, \ldots, x_{m-1} \in \mathbb{C}$, and $a_{m} \in A_{0}$ with $x_{0}+a_{0} \neq 0$ such that

$$
\left(x_{0}+a_{0}\right) a^{m}+\left(x_{1}+a_{1}\right) a^{m-1}+\cdots+\left(x_{m-1}+a_{m-1}\right) a+a_{m}=0 .
$$

Then $\theta_{\alpha}(a)$ is a root of the following non-zero polynomial:

$$
\left(x_{0}+\theta_{\alpha}\left(a_{0}\right)\right) X^{m}+\left(x_{1}+\theta_{\alpha}\left(a_{1}\right)\right) X^{m-1}+\cdots+\left(x_{m-1}+\theta_{\alpha}\left(a_{m-1}\right)\right) X+\theta_{\alpha}\left(a_{m}\right) .
$$

When we vary $\alpha \in S$, by the assumption, we will only obtain a finite number of such polynomials. Each such polynomial has at most $m$ roots in $D^{\#}$. So the set $\left\{\theta_{\alpha}(a): \alpha \in S\right\}$ is finite.

Remark. Let $\lambda$ be any infinite cardinal. Theorem 6.5 can be generalized by replacing pseudo-finiteness of $\left(P_{\alpha}\right)$ with the condition that "for each $a \in \bigcup_{\alpha \in S} P_{\alpha}$, the cardinality of $\left\{\alpha \in S: a \notin P_{\alpha}\right\}$ is strictly less than $\lambda$ ", and replacing (b) with the requirement that "the cardinality of $\left\{\theta_{\alpha}(a): \alpha \in S\right\}$ is strictly less than $\lambda$ for each $a \in A$ ". The above proof carries over with obvious modifications.

Theorem 6.7. Let $A$ be a commutative algebra, and let $\left(P_{\alpha}: \alpha \in S\right)$ be a pseudofinite family of non-modular prime ideals in $A$ indexed by a non-empty set $S$. Suppose that

$$
\left|A / \bigcap_{\alpha \in S} P_{\alpha}\right|=\mathfrak{c} .
$$

Then there exist a free ultrafilter $\mathcal{V}$ on $\mathbb{N}$ and homomorphisms $\varphi_{\alpha}: A \rightarrow c_{0} / \mathcal{V}$ for each $\alpha \in S$ such that:

(a) $\operatorname{ker} \varphi_{\alpha}=P_{\alpha}(\alpha \in S)$, and

(b) the set $\left\{\varphi_{\alpha}(a): \alpha \in S\right\}$ is finite for each $a \in A$.

Proof. First we find homomorphisms $\theta_{\alpha}$ from $A$ into $\left(\mathbb{C}^{\kappa} / \mathcal{U}\right)^{\circ}$ for some ultrapower $\mathbb{C}^{\kappa} / \mathcal{U}$, as in Theorem 6.5. Let $B$ be the subalgebra of $\left(\mathbb{C}^{\kappa} / \mathcal{U}\right)^{\circ}$ generated by all the images of $\theta_{\alpha}(\alpha \in S)$. Then $B$ is a non-unital integral domain. We also have

$$
|B|=\left|\bigcup_{\alpha \in S} \theta_{\alpha}(A)\right|=\left|\bigcup_{a \in A}\left\{\theta_{\alpha}(a): \alpha \in S\right\}\right|=\mathfrak{c} ;
$$

since, for each $b \in a+\bigcap_{\alpha \in S} P_{\alpha}$, we have

$$
\left\{\theta_{\alpha}(b): \alpha \in S\right\}=\left\{\theta_{\alpha}(a): \alpha \in S\right\} .
$$

Let $\psi: B \rightarrow c_{0} / \mathcal{V}$ be the embedding guaranteed by Theorem 6.1. We can then set $\varphi_{\alpha}=\psi \circ \theta_{\alpha}$ for each $\alpha \in S$.

Remark. For the objective of proving Theorems 7.2 and 7.4 in the next section, we only need the fact that the algebra $B$ in the previous proof is a non-unital integral domain of cardinality $\mathfrak{c}$, and so can be embedded into some radical Banach algebra. However, by using Theorem 6.1, we can replace $B$ by $c_{0} / \mathcal{V}$, which is more "concrete", so that the result is (at least theoretically) "nicer". 


\section{NORMing COMMUTATIVE SEMIPRIME ALGEBRAS AND THE CONVERSE OF THEOREM 4.5}

We now aim to generalize the result in [13] mentioned earlier on embedding nonunital integral domains of cardinality $\mathfrak{c}$ into (radical) Banach algebras. Specifically, we seek to construct an algebra norm on $A / \cap P_{\alpha}$, given a commutative algebra $A$ and a family of non-modular prime ideals $P_{\alpha}$ indexed by a set $S$ (we are mostly interested in the case where the index set $S$ is infinite). An "obvious" approach is to choose, assuming the Continuum Hypothesis as well as some "minor" cardinality condition, for each $\alpha \in S$, an algebra seminorm $p_{\alpha}$ on $A$ such that $\operatorname{ker} p_{\alpha}=P_{\alpha}$ (by the mentioned result), and then attempt to define an algebra seminorm $p$ on $A$ with kernel $\bigcap P_{\alpha}$ by setting

$$
p(a)=\sup _{\alpha \in S} p_{\alpha}(a) \quad(a \in A) .
$$

Unfortunately, unless the index set $S$ is a finite set, the above supremum can well be infinite. (In fact, when $S$ is infinite, for each $a \notin \bigcup P_{\alpha}$, there exists a family $\left\{p_{\alpha}: \alpha \in S\right\}$ such that $p(a)=\infty$.) However, the finiteness condition can be relaxed, yet this approach is still applicable if we can find a "nice" family of seminorms. Indeed, when the family $\left\{P_{\alpha}: \alpha \in S\right\}$ is pseudo-finite, because of the results in \$6. a family of seminorms $\left\{p_{\alpha}: \alpha \in S\right\}$ can be chosen such that $\left\{p_{\alpha}(a): \alpha \in S\right\}$ is only a finite set for each $a \in A$. Before going into details, we need to recall some definitions.

For the definition of universal algebras, see [6, Definition 5.7.8]. The important fact that we need is the existence of universal radical Banach algebras. For example, the integral domain $L^{1}\left(\mathbb{R}^{+}, \omega\right)$ is universal for each radical weight $\omega$ bounded near the origin [6, Theorem 5.7.25]. Indeed, the class of universal commutative radical Banach algebras has been characterized in [14] (see also [6, Theorem 5.7.28]). We also need the following theorem from [13] (see also [6, Theorem 5.7.11]); actually, we only need the particular case of $A=c_{0} / \mathcal{V}$.

Theorem $7.1(\mathrm{CH})$. Let $A$ be a non-unital integral domain of cardinality $\mathfrak{c}$, and let $L$ be a universal algebra. Then $A$ can be embedded into $L$.

Let $B$ be a Banach algebra, and let $S$ be an indexing set. Define $\ell^{\infty}(S, B)$ to be the Banach algebra of all bounded families $\left(b_{\alpha}: \alpha \in S\right)$ in $B$ under pointwise algebraic operations and the supremum norm.

Theorem $7.2(\mathrm{CH})$. Let $A$ be a commutative algebra, and let $\left(P_{\alpha}: \alpha \in S\right)$ be a pseudo-finite family of non-modular prime ideals in A such that

$$
\left|A / \bigcap_{\alpha \in S} P_{\alpha}\right|=\mathfrak{c} \text {. }
$$

Then there exist a commutative radical Banach algebra $R$ and a homomorphism $\theta: A \rightarrow R$ with $\operatorname{ker} \theta=\bigcap_{\alpha \in S} P_{\alpha}$.

Proof. Let $\varphi_{\alpha}: A \rightarrow c_{0} / \mathcal{V}(\alpha \in S)$ be the homomorphisms specified in Theorem 6.7 (see also the Remark after that theorem). Fix a universal commutative radical Banach algebra $R_{0}$. By Theorem 7.1, there is an embedding $\psi: c_{0} / \mathcal{V} \rightarrow R_{0}$. Consider the following map:

$$
\theta: A \rightarrow \prod_{\alpha \in S} R_{0}, a \mapsto\left(\left(\psi \circ \varphi_{\alpha}\right)(a): \alpha \in S\right)
$$


Then $\theta$ is a homomorphism with kernel $\bigcap P_{\alpha}$. We see, by Theorem 6.7, that the image of $\theta$ is in $\ell^{\infty}\left(S, R_{0}\right)$, and indeed is in its radical $R$. Thus $\theta: A \rightarrow R$ is the required homomorphism.

Corollary 7.3 $(\mathrm{CH})$. Let $A$ be a commutative semiprime algebra with $|A|=\mathfrak{c}$. Suppose that $\{0\}$ is the intersection of a pseudo-finite family of non-modular prime ideals. Then $A$ is normable.

When the family of prime ideals contains modular ones, the problem cannot be dealt with as smoothly. This is because a unital normed algebra must have a character, but there are unital algebras without any character. The situation becomes more complicated when we have infinitely many modular prime ideals.

The next result is the desired converse of Theorem 4.5.

Theorem $7.4(\mathrm{CH})$. Let $A$ be a commutative Banach algebra. Suppose that $I=$ $\bigcap_{\alpha \in S} P_{\alpha}$, where $\left(P_{\alpha}: \alpha \in S\right)$ is a pseudo-finite family of non-closed prime ideals in A satisfying

$$
|A / I|=\mathfrak{c} .
$$

Then there exists a commutative Banach algebra $B$ and a homomorphism $\theta: A \rightarrow B$ with $\mathcal{I}(\theta)=\operatorname{ker} \theta=I$. In the case where all the $P_{\alpha}$ are non-modular, we can choose $B$ to be radical.

In the case where the family $\left(P_{\alpha}: \alpha \in S\right)$ is intersection non-redundant, the ideal $I$ cannot be represented as an intersection of strictly fewer than $|S|$ prime ideals in $A$.

Proof. Fix a universal commutative radical Banach algebra $R_{0}$ such that $R_{0}$ is an integral domain. Note that, for each $\alpha \in S$, either $P_{\alpha}$ is non-modular or $P_{\alpha}$ is modular and contained in the kernel of a character of $A$. So, in both cases, by Theorem [7.1, $A / P_{\alpha}$ can be embedded in $R_{0}^{\#}$ (and even in $R_{0}$ if $P_{\alpha}$ is nonmodular). Therefore, for each finite number of prime ideals $P_{\alpha_{k}}(1 \leq k \leq n)$, there is a homomorphism from $A$ into $\prod_{k=1}^{n} R_{0}^{\#}$ with kernel $\bigcap_{k=1}^{n} P_{\alpha_{k}}$. We now consider the case where $S$ is infinite. In this case, we see that $P=\bigcup_{\alpha \in S} P_{\alpha}$ is either a prime ideal in $A$, or $A$ itself.

Case 1: $P=A$. For each $P_{\alpha}$, there is a homomorphism $\theta_{\alpha}$ from $A$ into $R_{0}^{\#}$ (into $R_{0}$ if $P_{\alpha}$ is non-modular) with kernel $P_{\alpha}$. Define

$$
\theta: A \rightarrow \prod_{\alpha \in S} R_{0}^{\#}, a \mapsto\left(\theta_{\alpha}(a)\right) .
$$

It is easily seen that the image of $\theta$ is contained in $\ell^{\infty}\left(S, R_{0}^{\#}\right)$, even in the radical of $\ell^{\infty}\left(S, R_{0}\right)$ if all $P_{\alpha}$ are non-modular.

Case 2: $P$ is a non-modular prime ideal in $A$. Then each $P_{\alpha}$ is non-modular. So, by Theorem 7.2, there exists a homomorphism $\theta: A \rightarrow R$ with $\operatorname{ker} \theta=I$, where $R$ is the radical of $\ell^{\infty}\left(S, R_{0}\right)$.

Case 3: $P$ is a modular prime ideal in $A$. Let $u \in A$ be a modular identity for $P$. Choose $a \notin P$. Then $(1-u) a \in P$, so $(1-u) a \in P_{\alpha}$ for all except finitely many $\alpha \in S$. For these exceptional indices $\alpha$ we can proceed as in the first paragraph of the proof, so that we can suppose that $(1-u) a \in P_{\alpha}(\alpha \in S)$. Since each $P_{\alpha}$ is a prime ideal, we see that $u$ is a (common) modular identity for all the $P_{\alpha}$. Thus $u$ is a modular identity for $I$. Let $M$ be a maximal modular ideal in $A$ containing $P$. Then $M$ has codimension 1 , that is $A=\mathbb{C} u \oplus M$. Since $P$ and each $P_{\alpha}$ are 
non-modular in $M$, by either Cases 1 or 2 above, there is a homomorphism from $M$ into $\ell^{\infty}\left(S, R_{0}\right)$ with kernel $I$. We can then extend this to a homomorphism from $A$ into $\ell^{\infty}\left(S, R_{0}^{\#}\right)$ by sending $u$ to $\mathbf{1}=(1,1, \ldots)$.

Thus, in each case, we can find a homomorphism

$$
\theta: A \rightarrow \ell^{\infty}\left(S, R_{0}^{\#}\right), a \mapsto\left(\theta_{\alpha}(a): \alpha \in S\right),
$$

where $\theta_{\alpha}: A \rightarrow R_{0}^{\#}$ is a homomorphism with kernel $P_{\alpha}(n \in \mathbb{N})$. Fix $a \in \mathcal{I}(\theta)$ and $\alpha \in S$. Then $a$ is also in the continuity ideal of $\theta_{\alpha}$. The map $\theta_{\alpha}$ has kernel $P_{\alpha}$, which is not closed, and so $\theta_{\alpha}$ is discontinuous. Therefore $\mathfrak{S}\left(\theta_{\alpha}\right) \neq\{0\}$. Since $\theta_{\alpha}(a) \mathfrak{S}\left(\theta_{\alpha}\right)=\{0\}$ and $R_{0}^{\#}$ is an integral domain, we must have $\theta_{\alpha}(a)=0$. Thus, $\mathcal{I}(\theta) \subset P_{\alpha}(\alpha \in S)$, and hence $\mathcal{I}(\theta) \subset I=\operatorname{ker} \theta \subset \mathcal{I}(\theta)$.

The last assertion follows from Lemma 3.5

Remark. Let $A$ be a commutative algebra, and let $\left(P_{\alpha}: \alpha \in S\right)$ be a family of prime ideals in $A$ which is pseudo-finite and intersection non-redundant. Suppose that we have

$$
\left|A / \bigcap_{\alpha \in S} P_{\alpha}\right|=\mathfrak{c}
$$

Then we must have $|S| \leq \mathfrak{c}$, and with the Continuum Hypothesis there are three possibilities left for the set $S$ : either $S$ is a finite set or $|S|=\aleph_{0}$ or $|S|=\aleph_{1}$.

Example 7.5 (Example 3.7 revisited, with $\mathrm{CH}$ ). Let $\mathfrak{F}_{S}$ and $Q_{\alpha}(\alpha \in S)$ as in Example 3.7. Suppose that $|S|$ is either $\aleph_{0}$ or $\aleph_{1}$. Then $\left|\mathfrak{F}_{S}\right|=\mathfrak{c}$. Thus, by Corollary 7.3 , the semiprime algebra $\mathfrak{F}_{S} / \bigcap_{\alpha \in S} Q_{\alpha}$, which is a priori without any obvious algebra norm, is normable.

Example 7.6 (Example 3.8 revisited, with $\mathrm{CH}$ ). Let $\mathcal{A}_{S}$ and $P_{\alpha}(\alpha \in S)$ be as in Example 3.8. Suppose that $|S| \leq \mathfrak{c}$. Applying Theorem 7.4, we can find a homomorphism from the commutative Banach algebra $\mathcal{A}_{S}$ into another Banach algebra whose continuity ideal is $\bigcap_{\alpha \in S} P_{\alpha}$, which is an intersection of $|S|$ prime ideals but not fewer. Thus, we have an example of a homomorphism whose continuity ideal is a semiprime ideal but not a finite intersection of prime ideals, in the case where $|S|$ is either $\aleph_{0}$ or $\aleph_{1}$.

\section{The Case $\mathcal{C}_{0}(\Omega)$ : Positive Results}

In this section, we shall consider two classes of spaces $\Omega$ for which Questions $A$ and $\mathrm{B}$ have a positive answer. First, applying the results of previous sections, we have the following reduction.

Theorem 8.1. Let $\Omega$ be a non-compact locally compact space.

(i) Let $\theta$ be a homomorphism from $\mathcal{C}_{0}(\Omega)$ into a radical Banach algebra $R$. Then either $\operatorname{ker} \theta$ is a finite intersection of non-modular prime ideals in $\mathcal{C}_{0}(\Omega)$, or $\mathcal{C}_{0}(\Omega)$ contains an intersection non-redundant pseudo-finite sequence of non-modular prime ideals.

(ii) $(\mathrm{CH})$ Suppose that $\left(P_{n}\right)$ is an intersection non-redundant pseudo-finite sequence of non-modular prime ideals in $\mathcal{C}_{0}(\Omega)$. Suppose further that

$$
\left|\mathcal{C}_{0}(\Omega) / \bigcap_{n=1}^{\infty} P_{n}\right|=\mathfrak{c} .
$$


Then there exists a homomorphism from $\mathcal{C}_{0}(\Omega)$ into a commutative radical Banach algebra with kernel $\bigcap_{n=1}^{\infty} P_{n}$, which is not a finite intersection of prime ideals.

Proof. (i) Since $\theta$ maps into a radical algebra, we see that $\operatorname{ker} \theta: f$ is non-modular for each $f \in \mathcal{C}_{0}(\Omega)$. The result then follows from Theorems 4.5 and 1.1.

(ii) This is a special case of Theorem 7.2 .

Remark.

1. A similar result for Question B follows from Theorems 4.5, 1.1(iv), and 7.4.

2. Let $I$ be the kernel (respectively, the continuity ideal) of a homomorphism from $\mathcal{C}_{0}(\Omega)$ into a radical Banach algebra (respectively, a Banach algebra). Denote by $\mathfrak{P}$ the set of prime ideals of the form $I: f$ for some $f \in \mathcal{C}_{0}(\Omega)$. With a little more effort, we can show, in this case, that each sequence of distinct prime ideals in $\mathfrak{P}$ contains an intersection non-redundant pseudo-finite subsequence. So, although $\mathfrak{P}$ is not always finite (as we shall see), it does have a "compactness type" property.

Lemma 8.2. Let $\Omega$ be a locally compact space. Let $\left(P_{\alpha}: \alpha \in S\right)$ be a pseudo-finite family of prime ideals in $\mathcal{C}_{0}(\Omega)$. Then $\bigcup_{\alpha \in S} P_{\alpha} \neq \mathcal{C}_{0}(\Omega)$. Furthermore, if $S$ is infinite, then $\bigcup_{\alpha \in S} P_{\alpha}$ is a prime ideal.

Proof. Suppose first that $S$ is infinite. Choose distinct elements $\alpha_{n}(n \in \mathbb{N})$ in $S$. For each $n \in \mathbb{N}$, choose $f_{n} \notin P_{\alpha_{n}}$; we can further suppose that

$$
0 \leq f_{n}(p) \leq \frac{1}{2^{n}} \quad(p \in \Omega) .
$$

Set $f=\sum_{n=1}^{\infty} f_{n}$. Then $f \in \mathcal{C}_{0}(\Omega)$, but $f \notin P_{\alpha_{n}}$ because $f \geq f_{n}(n \in \mathbb{N})$. By pseudo-finiteness of $\left(P_{\alpha}\right)$, we must have $f \notin \bigcup_{\alpha \in S} P_{\alpha}$. Hence $\bigcup_{\alpha \in S} P_{\alpha} \neq \mathcal{C}_{0}(\Omega)$.

The case where $S$ is finite can be proved similarly.

We have seen that our questions can be translated into an algebraic question of the structure of the algebra in consideration (subject to the Continuum Hypothesis in one of the directions). We now explain how Question A (and B) on $\mathcal{C}_{0}(\Omega)$ depends purely on the topological property of the underlying space $\Omega$.

Note that, for a pseudo-finite sequence of prime $z$-filters $\left(\mathcal{F}_{n}\right)$, the union $\bigcup_{n=1}^{\infty} \mathcal{F}_{n}$ is again a prime $z$-filter, and also that $\left(\mathbf{Z}^{-1}\left[\mathcal{F}_{n}\right]\right)$ is a pseudo-finite sequence of prime $z$-ideals in $\mathcal{C}(\Omega)$.

Lemma 8.3. Let $\Omega$ be a locally compact space. Let $\left(\mathcal{F}_{n}\right)$ be a pseudo-finite sequence of prime $z$-filters on $\Omega$. Then the following are equivalent:

(a) $\left(\mathcal{F}_{n}\right)$ is intersection non-redundant;

(b) $\mathcal{F}_{m} \not \subset \mathcal{F}_{n} \quad(m \neq n \in \mathbb{N})$;

(c) $\bigcap_{n \neq m} \mathcal{F}_{n} \not \subset \mathcal{F}_{m}$ for each $m \in \mathbb{N}$.

Proof. The proof is the same as that of Lemma 3.4.

By Lemmas 3.9] 3.10, and 8.2, we see that the question of the existence of an intersection non-redundant pseudo-finite sequence of prime ideals for $\mathcal{C}_{0}(\Omega)$ is equivalent to the same question for $\mathcal{C}\left(\Omega^{b}\right)$. Thus, we can pass freely between $\Omega$ and the compact space $\Omega^{b}$.

Let $\Omega$ be a locally compact space, and let $E$ be a closed subset of $\Omega$. We define

$$
I_{E}=\left\{f \in \mathcal{C}_{0}(\Omega): E \subset \mathbf{Z}(f)\right\} .
$$


Lemma 8.4. Let $\Omega$ be a compact space. Then the following are equivalent:

(a) there exists an intersection non-redundant pseudo-finite sequence of prime ideals $\left(P_{n}\right)$ in $\mathcal{C}(\Omega)$

(b) there exists an intersection non-redundant pseudo-finite sequence of prime $z$-filters $\left(\mathcal{F}_{n}\right)$ on $\Omega$;

(c) there exists an intersection non-redundant pseudo-finite sequence of prime $z$-ideals $\left(Q_{n}\right)$ in $\mathcal{C}(\Omega)$.

Proof. Obviously (b) $\Leftrightarrow(\mathrm{c}) \Rightarrow(\mathrm{a})$.

Now, we shall prove that (a) $\Rightarrow(\mathrm{b})$. For each $n \in \mathbb{N}$, set

$$
\mathcal{F}_{n}=\left\{Z \in \mathbf{Z}[\Omega]: I_{Z} \subset P_{n}\right\} .
$$

Then $\mathcal{F}_{n}$ is a prime $z$-filter (by [9, Theorem 4.22]).

Let $Z \in \mathbf{Z}[\Omega]$. Suppose that $Z \notin \mathcal{F}_{n_{i}}$, for some (increasing) sequence $\left(n_{i}\right)$. For each $i \in \mathbb{N}$, choose $f_{i} \in I_{Z} \backslash P_{n_{i}}$; we can further suppose that $0 \leq f_{i} \leq 1 / 2^{i}$. Define $f=\sum_{i=1}^{\infty} f_{i}$. Then $f \in I_{Z}$, but $f \notin P_{n_{i}}$, since $f \geq f_{i}(i \in \mathbb{N})$. By the pseudo-finiteness of $\left(P_{n}\right)$, we must have $f \notin P_{n}(n \in \mathbb{N})$. So $Z \notin \mathcal{F}_{n}(n \in \mathbb{N})$. This proves the pseudo-finiteness of $\left(\mathcal{F}_{n}\right)$.

Now assume toward a contradiction that $\mathcal{F}_{n_{0}} \subset \mathcal{F}_{m_{0}}$ for some $n_{0} \neq m_{0}$. Then

$$
Q:=\mathbf{Z}^{-1}\left[\mathcal{F}_{n_{0}}\right] \subset \mathbf{Z}^{-1}\left[\mathcal{F}_{m_{0}}\right] \subset P_{m_{0}} .
$$

Also we have $Q \subset P_{n_{0}}$. Since $Q$ is a prime ideal in $\mathcal{C}(\Omega)$, it follows that either $P_{m_{0}} \subset P_{n_{0}}$ or $P_{n_{0}} \subset P_{m_{0}}$, contradicting the intersection non-redundancy of $\left(P_{n}\right)$. Hence $\mathcal{F}_{n} \not \subset \mathcal{F}_{m}(n \neq m \in \mathbb{N})$. By Lemma 8.3, we conclude that $\left(\mathcal{F}_{n}\right)$ is intersection non-redundant.

Let $\Omega$ be a locally compact space. Suppose that $\left(P_{n}\right)$ is a pseudo-finite sequence of prime ideals in $\mathcal{C}_{0}(\Omega)$. Then $P=\bigcup_{n=1}^{\infty} P_{n}$ is again a prime ideal. Let $p \in \Omega^{b}$ be the unique support point for $P$. Then $p$ is also the support point for each $P_{n}$, and so we have

$$
J_{p} \subset P_{n} \subset P \subset M_{p} \quad(n \in \mathbb{N}) .
$$

Proposition 8.5. Let $\Omega$ be a locally compact space satisfying one of the following conditions:

(i) $\beta \Omega$ is an F-space, or

(ii) $\beta(\Omega \backslash\{p\})$ is an F-space for each non-P-point $p$ in $\Omega^{b}$.

Then $\mathcal{C}_{0}(\Omega)$ contains no intersection non-redundant pseudo-finite sequence of prime ideals.

Proof. Assume toward a contradiction that $\mathcal{C}_{0}(\Omega)$ contains an intersection nonredundant pseudo-finite sequence of prime ideals $\left(P_{n}\right)$.

(i) Since $\mathcal{C}_{0}(\Omega)$ is an ideal in $\mathcal{C}(\beta \Omega)$, by Lemmas 3.9 and 8.2 , we can find an intersection non-redundant pseudo-finite sequence $\left(Q_{n}\right)$ of prime ideals in $\mathcal{C}(\beta \Omega)$. As in the paragraph preceding this proposition, we see that $Q_{n}(n \in \mathbb{N})$ contains $J_{p}^{\beta \Omega}$ for some $p \in \beta \Omega$. Since $\beta \Omega$ is an F-space, it follows that $J_{p}^{\beta \Omega}$ is a prime ideal (see, for example, [6. Proposition 4.2.18]), and so $\left(Q_{n}\right)$, ordered by inclusion, must be a chain. This contradicts the intersection non-redundancy of $\left(Q_{n}\right)$.

(ii) Again, we have seen that, for some $p \in \Omega^{b}$,

$$
J_{p}^{\Omega} \subset P_{n} \subset M_{p}^{\Omega} \quad(n \in \mathbb{N}) .
$$


If $p$ is a P-point in $\Omega^{b}$, then $J_{p}^{\Omega}=M_{p}^{\Omega}$. So $P_{n}=J_{p}^{\Omega}(n \in \mathbb{N})$, contradicting the intersection non-redundancy of $\left(P_{n}\right)$. Hence $p$ is a non-P-point in $\Omega^{b}$. The ideal $M_{p}^{\Omega}$ can be naturally identified with $\mathcal{C}_{0}(\Omega \backslash\{p\})$. The desired contradiction then follows from (i).

Combining Theorems 1.1(iv), 4.5, and Proposition 8.5, we obtain the following theorem of [10].

Theorem 8.6. Let $\Omega$ be a locally compact space satisfying the hypothesis of Proposition 8.5. Then the continuity ideal of each homomorphism from $\mathcal{C}_{0}(\Omega)$ into another Banach algebra is always a finite intersection of prime ideals.

Next, we consider another class of locally compact spaces.

Proposition 8.7. Let $\Omega$ be a compact space. Suppose that $p \in \Omega \backslash \partial^{(\infty)} \Omega$. Then $\mathcal{C}(\Omega)$ does not contain an intersection non-redundant pseudo-finite sequence of prime ideals such that each $P_{n}$ is supported at the point $p$.

Proof. In the case where $\Omega$ has a finite limit level, let $l \in \mathbb{N}$ be such that $\partial^{(l)} \Omega=\emptyset$. For each $j \in \mathbb{Z}$ with $0 \leq j \leq l$, set $I_{j}=I_{\partial^{(j)} \Omega}$. Note that $I_{0}=\{0\}$ and $I_{l}=\mathcal{C}_{0}(\Omega)$. We see that, for each $j, I_{j} / I_{j-1}$ is isomorphic to $\mathcal{C}_{0}\left(U_{j}\right)$, where $U_{j}=\partial^{(j-1)} \Omega \backslash \partial^{(j)} \Omega$ is discrete and so satisfies condition (i) of Proposition 8.5. Thus each algebra $I_{j} / I_{j-1}$ does not contain an intersection non-redundant pseudo-finite sequence of prime ideals, and, applying Lemma 3.10 inductively, we deduce that neither does $I_{l}=\mathcal{C}_{0}(\Omega)$. The result holds in this case.

In general, assume toward a contradiction that $\mathcal{C}(\Omega)$ contains an intersection non-redundant pseudo-finite sequence $\left(P_{n}\right)$ of prime ideals such that each ideal is supported at $p$. Let $K$ be a compact neighbourhood of $p$ such that $K \cap \partial^{(\infty)} \Omega=\emptyset$. Then, since $I_{K} \subset J_{p} \subset P_{n}(n \in \mathbb{N})$, we see that $\mathcal{C}(\Omega) / I_{K}$, which is isomorphic to $\mathcal{C}(K)$, contains an intersection non-redundant pseudo-finite sequence of prime ideals. However, $K$ has a finite limit level, thus contradicting the previously proved case.

Theorem 8.8. Let $\Omega$ be a locally compact space. Denote by $p$ the point adjoined to $\Omega$ to obtain $\Omega^{b}$.

(i) Suppose that $p \notin \partial^{(\infty)}\left(\Omega^{b}\right)$. Then the kernel of each homomorphism from $\mathcal{C}_{0}(\Omega)$ into a radical Banach algebra is a finite intersection of non-modular prime ideals.

(ii) Suppose that $\Omega^{b}$ has finite limit level (i.e., $\left.\partial^{(\infty)}\left(\Omega^{b}\right)=\emptyset\right)$. Then each homomorphism from $\mathcal{C}_{0}(\Omega)$ into a Banach algebra is continuous on a finite intersection of prime ideals.

Proof. This follows from Theorem 8.1(i) and Proposition 8.7, see also the remark after Theorem 8.1 .

\section{The case $\mathcal{C}_{0}(\Omega)$ : Negative Results}

In this section, we show that, in the class of locally compact metrizable spaces, the only spaces for which the answer to Questions A and B is positive are those which satisfy the hypothesis of Theorem 8.8 .

First, we define two "prototype" spaces $\Xi_{0}$ and $\Xi_{1}$. Denote by $\infty$ the point adjoined to $\mathbb{N}$ to obtain its one-point compactification $\mathbb{N}^{b}$. The product space $\left(\mathbb{N}^{b}\right)^{\mathbb{N}}$ 
is a compact metrizable space. For abbreviation, we shall write $\left(n_{1}, n_{2}, \ldots, n_{k}\right)$ instead of $\left(n_{1}, n_{2}, \ldots, n_{k}, \infty, \infty, \ldots\right)$, where $n_{1}, \ldots, n_{k} \in \mathbb{N}^{b}$. Define $\Xi_{0}$ to be the subset of $\left(\mathbb{N}^{b}\right)^{\mathbb{N}}$ of all elements of the form $\left(n_{1}, n_{2}, \ldots, n_{k}\right)$, where $k \in \mathbb{Z}^{+}$and $n_{1}, n_{2}, \ldots, n_{k} \in \mathbb{N}$ are such that $k \leq n_{1}<n_{2}<\cdots<n_{k}$. Define $\Xi_{1}$ to be the subset of $\left(\mathbb{N}^{b}\right)^{\mathbb{N}}$ of all elements that can be written in the (not necessarily unique) abbreviated form as $\left(n_{1}, n_{2}, \ldots, n_{k}\right)$, where $k \in \mathbb{Z}^{+}$and $n_{1}, n_{2}, \ldots, n_{k} \in \mathbb{N}^{b}$ are such that $n_{i} \geq k(1 \leq i \leq k)$.

Lemma 9.1. (i) The sets $\Xi_{0} \subset \Xi_{1}$ are countable compact subspaces of $\left(\mathbb{N}^{b}\right)^{\mathbb{N}}$.

(ii) There exists a continuous embedding of $\Xi_{1}$ into $\Xi_{0}$; it follows automatically that the point $(\infty, \infty, \ldots)$ is mapped to itself.

Proof. Part (i) is routine.

We now prove part (ii). First, we see that the space $\Xi_{0}$ is homeomorphic to the subspace

$$
\Delta:=\{0\} \cup\left\{\sum_{i=1}^{k} 2^{-n_{i}}: k, n_{1}, n_{2}, \ldots, n_{k} \in \mathbb{N} \text { and } k \leq n_{1}<\cdots<n_{k}\right\}
$$

of $[0,1]$. Next, for each $i \in \mathbb{N}$, choose an increasing sequence $\left(r_{i j}: j \in \mathbb{N}\right)$ in $\mathbb{N}$ such that these sequences are pairwise disjoint; furthermore, we can suppose that $r_{i j} \geq j(i, j \in \mathbb{N})$. For convenience, for each $i \in \mathbb{N}$, set $r_{i \infty}=\infty$. Also, our convention is that $2^{-\infty}=0$. Define a map $\tau$ from $\Xi_{1}$ into $[0,1]$ as follows: for each $\left(n_{1}, n_{2}, \ldots\right) \in \Xi_{1}$, set

$$
\tau\left(n_{1}, n_{2}, \ldots\right)=\sum_{i=1}^{\infty} 2^{-r_{i n_{i}}} .
$$

We see that $\tau$ is well-defined, injective and continuous. It can be checked that $\tau$ actually maps $\Xi_{1}$ into $\Delta$. The result then follows.

Lemma 9.2. Let $\Omega$ be a locally compact metrizable space. Suppose that there exists a point $p \in \partial^{(\infty)}\left(\Omega^{b}\right)$. Then there exists a continuous embedding $\iota: \Xi_{1} \hookrightarrow \Omega^{b}$ such that $\iota(\infty, \infty, \ldots)=p$.

Remark. It follows that $\Xi_{1}$ is homeomorphic to the subspace $\iota\left(\Xi_{1}\right)$ of $\Omega^{b}$.

Proof of Lemma 9.2. Fix a metric on $\Omega$. In this proof, we shall denote by $\mathrm{B}_{x}$ an open ball in $\Omega$ centered at a point $x \in \Omega$ with some positive radius, whose exact value is not important for our purpose. We first aim to embed $\Xi_{0}$ into $\Omega^{b}$.

We claim that there exists a sequence $\left(\iota_{1}(n)\right)$ of distinct elements in $\Omega \backslash\{p\}$ such that $\lim _{n \rightarrow \infty} \iota_{1}(n)=p$ and that $\iota_{1}(n) \in \partial^{(n-1)}\left(\Omega^{b}\right)(n \in \mathbb{N})$. The proof of this is divided into the three following cases:

Case 1: $p \in \Omega$. Since $\Omega$ is metrizable and $p \in \partial^{(\infty)}\left(\Omega^{b}\right)$, this case is obvious.

Case 2: $p$ is the point adjoined to $\Omega$ to obtain $\Omega^{b}$, and $p$ is a limit point of $\partial^{(\infty)}\left(\Omega^{b}\right)$. Then, since $\Omega \cap \partial^{(\infty)}\left(\Omega^{b}\right)$ is non-compact and metrizable, it is not sequentially compact. Choose a sequence $\left(\iota_{1}(n)\right)$ with no convergent subsequences in $\Omega \cap \partial^{(\infty)}\left(\Omega^{b}\right)$. Then $\lim _{n \rightarrow \infty} \iota_{1}(n)=p$ in $\Omega^{b}$.

Case 3: $p$ is the point adjoined to $\Omega$ to obtain $\Omega^{b}$, and $p$ is an isolated point in $\partial^{(\infty)}\left(\Omega^{b}\right)$. Choose a compact neighbourhood $K$ of $p$ such that $K \cap \partial^{(\infty)}\left(\Omega^{b}\right)=\{p\}$. We see that $\partial^{(\infty)} K=\{p\}$. For each $n \in \mathbb{N}$, we choose an element

$$
\iota_{1}(n) \in \partial^{(n-1)} K \backslash\{p\} \subset \Omega \cap \partial^{(n-1)}\left(\Omega^{b}\right) .
$$


Then each limit point of $\left\{\iota_{1}(n): n \in \mathbb{N}\right\}$ must be in $\bigcap \partial^{(n)} K=\partial^{(\infty)} K$. Therefore we deduce that $\lim _{n \rightarrow \infty} \iota_{1}(n)=p$.

Thus the claim holds.

Now we choose a sequence of pairwise disjoint balls $\left(\mathrm{B}_{\iota_{1}(n)}\right)$ such that each $\mathrm{B}_{\iota_{1}(n)}$ has radius at most $2^{-n}$ and does not contain $p$. We observe that if, for each $n \in \mathbb{N}$, we choose $x_{n} \in \mathrm{B}_{\iota_{1}(n)}$, then we still have $\lim x_{n}=p$.

Let $k \in \mathbb{N}$. Assuming that, for each $n_{1}, n_{2}, \ldots, n_{k} \in \mathbb{N}$ with $n_{1} \geq k$, we already have a ball

$$
\mathrm{B}_{\iota_{1}\left(n_{1}, \ldots, n_{k}\right)} \text { with } \iota_{1}\left(n_{1}, \ldots, n_{k}\right) \in \partial^{\left(n_{1}-k\right)} \Omega .
$$

We continue the construction inductively as follows. If $n_{1}>k$, then, for each $n \in \mathbb{N}$, we can choose a ball $\mathrm{B}_{\iota_{1}\left(n_{1}, \ldots, n_{k}, n\right)}$ contained in $\mathrm{B}_{\iota_{1}\left(n_{1}, \ldots, n_{k}\right)}$, not containing $\iota_{1}\left(n_{1}, \ldots, n_{k}\right)$, and whose center is of distance less than $2^{-n}$ from the point $\iota_{1}\left(n_{1}, \ldots, n_{k}\right)$, such that all these new balls are disjoint; furthermore, we can assume that all the new points

$$
\iota_{1}\left(n_{1}, \ldots, n_{k}, n\right) \in \partial^{\left(n_{1}-k-1\right)} \Omega \quad(n \in \mathbb{N}) .
$$

Finally, set $\iota_{1}(\infty, \infty, \ldots)=p$.

Obviously, $\Xi_{0}$ is contained in the domain of definition of $\iota_{1}$. Consider the restriction $\iota_{2}: \Xi_{0} \rightarrow \Omega$ of $\iota_{1}$. We see that $\iota_{2}$ is injective and continuous. The result then follows by applying Lemma 9.1 .

Let $\Omega$ be a compact space, and let $\mathcal{F}$ be a prime $z$-filter on $\Omega$. Then we say that $\mathcal{F}$ is supported at a point $p$ if $p \in Z$ for each $Z \in \mathcal{F}$. There always exists a unique support point for each prime $z$-filter (on a compact space).

Lemma 9.3. There exists an intersection non-redundant pseudo-finite sequence of prime $z$-filters on $\Xi_{1}$ such that each $z$-filter is supported at the point $(\infty, \infty, \ldots)$.

Proof. For each $n \in \mathbb{N}$, define $f_{n} \in \mathcal{C}\left(\Xi_{1}\right)$ by setting $f_{n}\left(j_{1}, j_{2}, \ldots\right)=2^{-j_{n}}$ for each $\left(j_{1}, j_{2}, \ldots\right) \in \Xi_{1}$. Thus

$$
\mathbf{Z}\left(f_{n}\right)=\left\{\left(j_{1}, j_{2}, \ldots\right) \in \Xi_{1}: j_{n}=\infty\right\}
$$

Let $\mathcal{F}$ to be the $z$-filter generated by all $\mathbf{Z}\left(f_{m} f_{n}\right)(m, n \in \mathbb{N}, m \neq n)$. Then define $I=\mathbf{Z}^{-1}[\mathcal{F}]$. We claim that $I$ is extendible with respect to $\left(f_{n}\right)$ (see Definition 3.11).

Obviously, $f_{m} f_{n} \in I(m \neq n)$. We see that $\mathbf{Z}\left(f_{n}^{k}\right)=\mathbf{Z}\left(f_{n}\right) \notin \mathcal{F}$, and so $f_{n}^{k} \notin I$ $(n, k \in \mathbb{N})$. Now suppose that $g \in \mathcal{C}\left(\Xi_{1}\right)$ such that $g f_{n_{0}}^{k_{0}} \in I$ for some $n_{0}, k_{0} \in \mathbb{N}$. Then $\mathbf{Z}(g) \cup \mathbf{Z}\left(f_{n_{0}}\right) \in \mathcal{F}$. Therefore, there exist $n_{1}, n_{2}, \ldots, n_{k} \in \mathbb{N} \backslash\left\{n_{0}\right\}$ such that

$$
\mathbf{Z}(g) \cup \mathbf{Z}\left(f_{n_{0}}\right) \supset \bigcap_{i=1}^{k} \mathbf{Z}\left(f_{n_{i}}\right) .
$$

This and the closedness of $\mathbf{Z}(g)$ imply that

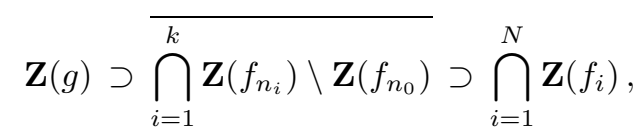

where $N=\max \left\{n_{0}, n_{1}, \ldots, n_{k}\right\}$. So, for each $n \geq N+1$, we have $g f_{n} \in I$. Hence the claim holds.

The proof is completed by applying Proposition 3.12 and Lemma 8.4 
Remark. We can prove a result on constructing pseudo-finite sequence of prime $z$-filters similar to Proposition 3.12, and then use it directly in the previous proof, so that we do not need to refer to Lemma 8.4

Recall that when $p$ is the point adjoined to $\Omega$ to obtain $\Omega^{b}$, a prime ideal $P$ in $\mathcal{C}_{0}(\Omega)$ is supported at $p$ means that $P$ is non-modular.

Proposition 9.4. Let $\Omega$ be a locally compact metrizable space. Suppose that $p \in$ $\partial^{(\infty)}\left(\Omega^{b}\right)$. Then there exists an intersection non-redundant pseudo-finite sequence $\left(\mathcal{F}_{n}\right)$ of prime $z$-filters on $\Omega^{b}$, where each $z$-filter is supported at $p$. Moreover, by setting $P_{n}=\mathcal{C}_{0}(\Omega) \cap \boldsymbol{Z}_{\Omega^{b}}^{-1}\left[\mathcal{F}_{n}\right]$, we obtain an intersection non-redundant pseudofinite sequence of prime ideals in $\mathcal{C}_{0}(\Omega)$, each of which is supported at $p$, such that

$$
\left|\mathcal{C}_{0}(\Omega) / \bigcap_{n=1}^{\infty} P_{n}\right|=\mathfrak{c} .
$$

Proof. This follows from Lemmas $9.2,9.3,8.4$ and the fact that $\left|\mathcal{C}\left(\Xi_{1}\right)\right|=\mathfrak{c}$.

The next theorem and its corollaries provide the negative answer to Questions $\mathrm{A}$ and $\mathrm{B}$.

Theorem 9.5 $(\mathrm{CH})$. Let $\Omega$ be a locally compact metrizable space. Denote by $p$ the point adjoined to $\Omega$ to obtain $\Omega^{b}$.

(i) Suppose that $p \in \partial^{(\infty)}\left(\Omega^{b}\right)$. Then there exists a homomorphism $\theta$ from $\mathcal{C}_{0}(\Omega)$ into a radical Banach algebra such that the kernel of $\theta$ is not a finite intersection of prime ideals.

(ii) Suppose that $\Omega^{b}$ has an infinite limit level (i.e., $\left.\partial^{(\infty)}\left(\Omega^{b}\right) \neq \emptyset\right)$. Then there exists a homomorphism $\theta$ from $\mathcal{C}_{0}(\Omega)$ into a Banach algebra such that the continuity ideal of $\theta$ is not a finite intersection of prime ideals.

Proof. This follows from Proposition 9.4 and Theorem 7.4 .

Note that each uncountable compact metrizable space $\Omega$ has an infinite limit level. For example, this is the case for the closed unit interval $[0,1]$.

Corollary 9.6 $(\mathrm{CH})$. There exists a homomorphism $\theta$ from $\mathcal{C}_{0}(\mathbb{R})$ into a radical Banach algebra such that the kernel of $\theta$ is not a finite intersection of prime ideals.

There are many countable compact metrizable spaces with infinite limit level. We note down the following specific example. Recall that in the proof of Lemma 9.1. we define the following countable compact subset of $[0,1]$ :

$$
\Delta=\{0\} \cup\left\{\sum_{i=1}^{k} 2^{-n_{i}}: k, n_{1}, n_{2}, \ldots, n_{k} \in \mathbb{N} \text { and } k \leq n_{1}<\cdots<n_{k}\right\} .
$$

Corollary $9.7(\mathrm{CH})$. There exists a homomorphism $\theta$ from $\mathcal{C}_{0}(\Delta \backslash\{0\})$ into a radical Banach algebra such that the kernel of $\theta$ is not a finite intersection of prime ideals.

\section{LOCALLY COMPACT GROUPS} $G$.

In this final section, we consider the algebra $\mathcal{C}_{0}(G)$ for a locally compact group 
Lemma 10.1. Let $G$ be a non-discrete locally compact group satisfying either one of the following conditions:

(i) $G$ is Abelian, or

(ii) $G$ is not totally disconnected.

Then $G$ contains a non-discrete metrizable closed subgroup.

Proof. For the case where $G$ is both compact and Abelian, see [22, Theorem 7].

In the case where $G$ is totally disconnected and Abelian, by [16, Theorem 7.7], $G$ contains a compact open subgroup. The result then follows from the previous case.

We now suppose that $G$ is not totally disconnected. Let $G_{1}$ be the connected component of the identity e of $G$. Then $G_{1}$ is a non-discrete closed normal subgroup of $G$. By Iwasawa structure theorem ([21, p. 118]), $G_{1}$ contains a closed subgroup homeomorphic to $\mathbb{R}^{n}$ for some $n \in \mathbb{Z}^{+}$and a compact subgroup $K$ such that $G_{1}$ is homeomorphic to $\mathbb{R}^{n} \times K$. If $n>0$, then we are done.

Now suppose that $n=0$, i.e., $G_{1}$ is compact. (In this case, for the purpose of proving the next lemma, we note that $G_{1}$ is contained in an open compact subgroup of $G$.) Let $G_{2}$ be a maximal connected Abelian subgroup of $G_{1}$. Then $G_{2}$ is closed, and, by [17, Theorem 9.32], $G_{2}$ is maximal among the Abelian subgroup of $G_{1}$. Therefore $G_{2} \neq\{\mathbf{e}\}$. The result, again, follows from the first case.

Lemma 10.2. Let $G$ be a non-discrete locally compact group. Then $G$ contains a metrizable closed subset $\Omega$ such that $\Omega$ has no isolated point. If, furthermore, $G$ is non-compact, then $\Omega$ can be further required to be non-compact either.

Proof. For the first assertion, by Lemma 10.1(ii), we only need to consider the case where $G$ is totally disconnected. In this case, by [16, Theorems 7.7 and 9.15], $G$ contains a compact open subgroup, where each such subgroup is homeomorphic to a space $\{0,1\}^{\kappa}$ for some infinite cardinal $\kappa$, and, in turn, each such space contains a copy of the Cantor set.

For the second assertion, we see that, in each case where our previously constructed set $\Omega$ is compact, $\Omega$ is contained in a compact open subgroup, say $H$, of $G$. In these cases, since $G$ is non-compact, we can choose a sequence $\left(x_{n}\right)$ in $G$ such that $x_{n} H(n \in \mathbb{N})$ are distinct elements of $G / H$; we can then replace $\Omega$ by $\bigcup_{n=1}^{\infty} x_{n} \Omega$.

Proposition 10.3. Let $G$ be a non-discrete locally compact group. Then there exists an intersection non-redundant pseudo-finite sequence of prime $z$-filters $\left(\mathcal{F}_{n}\right)$ on $G^{b}$. Furthermore, if $G$ is non-compact, we can require each $\mathcal{F}_{n}$ to be supported at the adjoined point (the point at infinity).

Proof. This follows from Proposition 9.4 and Lemma 10.2

Theorem 10.4. Let $G$ be a non-discrete locally compact group. Then:

(i) there exists an intersection non-redundant pseudo-finite sequence of prime ideals $\left(P_{n}\right)$ in $\mathcal{C}_{0}(G)$ with $\left|\mathcal{C}_{0}(G) / \cap_{n=1}^{\infty} P_{n}\right|=\mathfrak{c}$;

(ii) $(\mathrm{CH})$ there exists a homomorphism $\theta$ from $\mathcal{C}_{0}(G)$ into another Banach algebra such that the continuity ideal of $\theta$ is not a finite intersection of prime ideals.

Proof. These follow from Proposition 10.3 and Theorem 7.4 . 
Theorem 10.5. Let $G$ be a non-discrete non-compact locally compact group. Then:

(i) there exists an intersection non-redundant pseudo-finite sequence of nonmodular prime ideals $\left(P_{n}\right)$ in $\mathcal{C}_{0}(G)$ with $\left|\mathcal{C}_{0}(G) / \bigcap_{n=1}^{\infty} P_{n}\right|=\mathfrak{c}$;

(ii) $(\mathrm{CH})$ there exists a homomorphism $\theta$ from $\mathcal{C}_{0}(G)$ into a radical Banach algebra such that the kernel of $\theta$ is not a finite intersection of prime ideals.

Proof. These follow from Proposition 10.3 and Theorem 8.1 .

For each locally compact Abelian group $G$, denote by $\widehat{G}$ its dual group. For each $f \in L^{1}(G)$, denote by $\hat{f}$ the Fourier transform of $f$. Then

$$
f \mapsto \hat{f}, \quad L^{1}(G) \rightarrow \mathcal{C}_{0}(\widehat{G}),
$$

is a continuous homomorphism.

Lemma 10.6. Let $G$ be a locally compact Abelian group. Let $E$ be a closed subset of $\widehat{G}$ and let $K$ be a $\sigma$-compact subset of $\widehat{G}$ such that $E \cap K=\emptyset$. Then there exists $f \in L^{1}(G)$ such that $\hat{f}=0$ on $E$ but $\hat{f}$ never vanishes on $K$.

Proof. Let $K=\bigcup_{n=1}^{\infty} K_{n}$, where each $K_{n}$ is compact. By [6, Lemma 4.5.16], for each $n \in \mathbb{N}$, we can find $f_{n} \in L^{1}(G)$ such that $\hat{f}_{n}=0$ on $E$, such that $\hat{f}_{n}$ never vanishes on $K_{n}$, and such that $\hat{f}_{n} \geq 0$ on $\widehat{G}$. We can further suppose that $\left\|f_{n}\right\|_{1} \leq 2^{-n}$. Set $f=\sum_{n=1}^{\infty} f_{n}$. Then $f \in L^{1}(G)$ is the desired function.

Theorem 10.7. Let $G$ be a non-compact locally compact Abelian group. Then:

(i) there exists an intersection non-redundant pseudo-finite sequence of prime ideals $\left(P_{n}\right)$ in $L^{1}(G)$ with $\left|L^{1}(G) / \bigcap_{n=1}^{\infty} P_{n}\right|=\mathfrak{c}$;

(ii) $(\mathrm{CH})$ there exists a homomorphism $\theta$ from $L^{1}(G)$ into another Banach algebra such that $\mathcal{I}(\theta)=\operatorname{ker} \theta$ is a semiprime ideal but not a finite intersection of prime ideals.

Proof. Since $G$ is non-compact, the dual $\widehat{G}$ is non-discrete. Let $\left(\mathcal{F}_{n}\right)$ be an intersection non-redundant pseudo-finite sequence of prime $z$-filters on $\widehat{G}^{b}$ as specified in Proposition 10.3. For each $n \in \mathbb{N}$, set $Q_{n}=\mathcal{C}_{0}(\widehat{G}) \cap \mathbf{Z}_{\widehat{G}^{b}}^{-1}\left[\mathcal{F}_{n}\right]$. Then $\left(Q_{n}\right)$ is an intersection non-redundant pseudo-finite sequence of prime ideals in $\mathcal{C}_{0}(\widehat{G})$.

Denote by $p$ the point adjoined to $\widehat{G}$ to obtain $\widehat{G}^{b}$. We see that, for each $n \in \mathbb{N}$,

$$
Q_{n}=\left\{h \in \mathcal{C}_{0}(\widehat{G}): \mathbf{Z}_{\widehat{G}}(h) \cup\{p\} \in \mathcal{F}_{n}\right\} .
$$

Define, for each $n \in \mathbb{N}$,

$$
P_{n}=\left\{f \in L^{1}(G): \hat{f} \in Q_{n}\right\}=\left\{f \in L^{1}(G): \mathbf{Z}_{\widehat{G}}(\hat{f}) \cup\{p\} \in \mathcal{F}_{n}\right\} ;
$$

we obtain a pseudo-finite sequence $\left(P_{n}\right)$ of prime ideals in $L^{1}(G)$.

For each $n \in \mathbb{N}$, choose $g_{n} \in \bigcap_{i \neq n} Q_{i} \backslash Q_{n}$. By Lemma 10.6, there exists $f_{n} \in L^{1}(G)$ such that $\mathbf{Z}_{\widehat{G}}\left(\hat{f}_{n}\right)=\mathbf{Z}_{\widehat{G}}\left(g_{n}\right)$. Then we see that $f_{n} \in \bigcap_{i \neq n} P_{i} \backslash P_{n}$ $(n \in \mathbb{N})$. Hence $\left(P_{n}\right)$ is intersection non-redundant. The cardinality statement is obvious from the construction.

Part (ii) then follows from Theorem 7.4

Remark. In the preceding proof, by Lemma 10.6 if the ideal $Q_{n}$ is non-modular, then so is the ideal $P_{n}$. Therefore, if, furthermore, the topology on $G$ is nondiscrete, then we can further require each $P_{n}$ to be non-modular and the range of the homomorphism $\theta$ to be radical. 
For each discrete space $G$, there exists no intersection non-redundant pseudofinite sequence of prime ideals in $\mathcal{C}_{0}(G)$. However, this does not answer the following.

Question 10.8. Does there exist an intersection non-redundant pseudo-finite sequence of prime ideals in $L^{1}(G)$, for each compact Abelian group $G$ ?

\section{ACKNOWLEDGEMENTS}

The author would like to thank his supervisor, Professor H. Garth Dales, for all the help and encouragement. This work was undertaken while the author was studying for a Ph.D. at the University of Leeds, supported financially by the ORS, the School of Mathematics, and the University of Leeds.

\section{REFERENCES}

[1] W. G. Bade and P. C. Curtis, Jr., Homomorphisms of commutative Banach algebras, Amer. J. Math. 82 (1960), 589-608. MR0117577 (22:8354)

[2] J. Cusack, Automatic continuity and topologically simple radical Banach algebras, J. London Math. Soc. (2) 16 (1977), 493-500. MR0461136 (57:1121)

[3] H. G. Dales, The uniqueness of the functional calculus, Proc. London Math. Soc. (3) 27 (1973), 638-648. MR0333738 (48:12062)

[4] _ Automatic continuity: a survey, Bull. London Math. Soc. 10 (1978), 129-183. MR500923 (80c:46053)

[5] - A discontinuous homomorphism from $C(X)$, Amer. J. Math. 101 (1979), 647-734. MR.533196 (81g:46066)

[6] - Banach algebras and automatic continuity, London Mathematical Society Monographs, vol. 24, The Clarendon Press, Oxford, 2000. MR1816726 (2002e:46001)

[7] H. G. Dales and R. J. Loy, Prime ideals in algebras of continuous functions, Proc. Amer. Math. Soc. 98 (1986), 426-430. MR857934 (87j:46098)

[8] H. G. Dales and W. H. Woodin, An introduction to independence for analysts, London Mathematical Society Lecture Note Series, vol. 115, Cambridge University Press, Cambridge, 1987. MR942216 (90d:03101)

[9] _ Super-real fields: totally ordered fields with additional structure, London Mathematical Society Monographs, vol. 14, The Clarendon Press, Oxford, 1996. MR.1420859 (98b:12005)

[10] J. R. Esterle, Seminormes sur $C(K)$, Proc. London Math. Soc. (3) 36 (1978), 27-45. MR 0482215 (58:2297)

[11] Sur l'existence d'un homomorphisme discontinu de $C(K)$, Proc. London Math. Soc. (3) 36 (1978), 46-58. MR0482217 (58:2299)

[12] Injection de semi-groupes divisibles dans des algèbres de convolution et construction d'homomorphismes discontinus de $C(K)$, Proc. London Math. Soc. (3) 36 (1978), 59-85. MR0482218(58:2300)

[13] - Homomorphismes discontinus des algèbres de Banach commutatives séparables, Studia Math. 66 (1979), 119-141. MR565154 (81m:46067)

[14] Universal properties of some commutative radical Banach algebras, J. Reine Angew. Math. 321 (1981), 1-24. MR597976 (82i:46078)

[15] L. Gillman and M. Jerison, Rings of continuous functions, The University Series in Higher Mathematics, D. Van Nostrand Co., Inc., Princeton, New Jersey-Toronto-London-New York, 1960. MR0116199 (22:6994)

[16] E. Hewitt and K. A. Ross, Abstract harmonic analysis. Vol. I: Structure of topological groups. Integration theory, group representations, Die Grundlehren der mathematischen Wissenschaften, Bd. 115, Academic Press Inc., Publishers, New York, 1963. MR0156915 (28:158)

[17] K. H. Hofmann and S. A. Morris, The structure of compact groups, de Gruyter Studies in Mathematics, vol. 25, Walter de Gruyter \& Co., Berlin, 1998. MR.1646190 (99k:22001)

[18] B. E. Johnson, Norming $C(\Omega)$ and related algebras, Trans. Amer. Math. Soc. 220 (1976), 37-58. MR0415326 (54:3415)

[19] I. Kaplansky, Normed algebras, Duke Math. J. 16 (1949), 399-418. MR0031193 (11:115d) 
[20] K. Kendig, Elementary algebraic geometry, Springer-Verlag, New York, 1977, Graduate Texts in Mathematics, No. 44. MR0447222 (56:5537)

[21] S. A. Morris, Pontryagin duality and the structure of locally compact abelian groups, Cambridge University Press, Cambridge, 1977, London Mathematical Society Lecture Note Series, No. 29. MR0442141 (56:529)

[22] W. Rudin, Averages of continuous functions on compact spaces, Duke Math. J. 25 (1958), 197-204. MR0098313 (20:4774)

[23] A. M. Sinclair, Homomorphisms from $C_{0}(R)$, J. London Math. Soc. (2) 11 (1975), 165-174. MR0377517 (51:13689)

[24] Automatic continuity of linear operators, Cambridge University Press, Cambridge, 1976, London Mathematical Society Lecture Note Series, No. 21. MR0487371 (58:7011)

[25] J. L. Taylor, Several complex variables with connections to algebraic geometry and Lie groups, Graduate Studies in Mathematics, vol. 46, American Mathematical Society, Providence, RI, 2002. MR1900941 (2004b:32001)

Department of Pure Mathematics, University of Leeds, Leeds LS2 9JT, England

E-mail address: hung@maths.leeds.ac.uk

Current address: Department of Mathematical and Statistical Sciences, University of Alberta, Edmonton, Alberta, Canada T6G 2E1

E-mail address: hlpham@math.ualberta.ca 